\title{
Dynamical cloud formation traced by atomic and molecular gas $\star$
}

\author{
H. Beuther ${ }^{1}$, Y. Wang ${ }^{1}$, J. Soler ${ }^{1}$, H. Linz ${ }^{1}$, J. Henshaw ${ }^{1}$, E. Vazquez-Semadeni' ${ }^{2}$, G. Gomez ${ }^{2}$, S. Ragan ${ }^{3}$, \\ Th. Henning ${ }^{1}$, S. C. O. Glover ${ }^{4}$, M.-Y. Lee ${ }^{5}$, and R. Güsten ${ }^{6}$ \\ ${ }^{1}$ Max Planck Institute for Astronomy, Königstuhl 17, 69117 Heidelberg, Germany \\ e-mail: beuther@mpia.de \\ ${ }^{2}$ Instituto de Radioastronomía y Astrofísica, Universidad Nacional Autonoma de Mexico, Morelia, Michoacan 58089, Mexico \\ ${ }^{3}$ School of Physics and Astronomy, Cardiff University, Queen's Buildings, The Parade, Cardiff CF24 3AA, UK \\ ${ }^{4}$ University of Heidelberg, Institute for Theoretical Astrophysics, Albert-Ueberle-Str. 2, 69120 Heidelberg, Germany \\ ${ }^{5}$ Korea Astronomy and Space Science Institute, 776 Daedeok-daero, 34055 Daejeon, Republic of Korea \\ ${ }^{6}$ Max-Planck-Institut für Radioastronomie, Auf dem Hügel 69, 53121 Bonn, Germany
}

Received 13 March 2020 / Accepted 10 April 2020

\begin{abstract}
Context. Atomic and molecular cloud formation is a dynamical process. However, kinematic signatures of these processes are still observationally poorly constrained.

Aims. We identify and characterize the cloud formation signatures in atomic and molecular gas.

Methods. Targeting the cloud-scale environment of the prototypical infrared dark cloud G28.3, we employed spectral line imaging observations of the two atomic lines $\mathrm{HI}$ and $[\mathrm{CI}]$ as well as molecular lines observations in ${ }^{13} \mathrm{CO}$ in the $1-0$ and $3-2$ transitions. The analysis comprises investigations of the kinematic properties of the different tracers, estimates of the mass flow rates, velocity structure functions, a histogram of oriented gradients (HOG) study, and comparisons to simulations.

Results. The central infrared dark cloud (IRDC) is embedded in a more diffuse envelope of cold neutral medium traced by HI selfabsorption and molecular gas. The spectral line data as well as the HOG and structure function analysis indicate a possible kinematic decoupling of the $\mathrm{HI}$ from the other gas compounds. Spectral analysis and position-velocity diagrams reveal two velocity components that converge at the position of the IRDC. Estimated mass flow rates appear rather constant from the cloud edge toward the center. The velocity structure function analysis is consistent with gas flows being dominated by the formation of hierarchical structures.

Conclusions. The observations and analysis are consistent with a picture where the IRDC G28.3 is formed at the center of two converging gas flows. While the approximately constant mass flow rates are consistent with a self-similar, gravitationally driven collapse of the cloud, external compression (e.g., via spiral arm shocks or supernova explosions) cannot be excluded yet. Future investigations should aim at differentiating the origin of such converging gas flows.
\end{abstract}

Key words. ISM: clouds - ISM: kinematics and dynamics - ISM: structure - ISM: general - evolution - stars: formation

\section{Introduction}

Molecular clouds are formed out of the atomic phase of the interstellar medium (ISM). While models detailing the transition from the atomic to molecular phase exist (e.g., Franco \& Cox 1986; Hartmann et al. 2001; Bergin et al. 2004; Krumholz et al. 2008, 2009; Sternberg et al. 2014; Bialy et al. 2017), the observational constraints of the molecular cloud formation processes are still not properly characterized. Of particular importance is the dynamic state of the clouds, i.e., whether they are dominated by converging gas flows that could create overdensities important for the atomic-to-molecular gas conversion (e.g., Koyama \& Inutsuka 2000; Audit \& Hennebelle 2005, 2010; Vázquez-Semadeni et al. 2011, 2019; Heitsch et al. 2005, 2006; Heitsch \& Hartmann 2008; Banerjee et al. 2009; Clark et al. 2012; Gómez \& Vázquez-Semadeni 2014; Motte et al. 2014; Heiner et al. 2015; Henshaw et al. 2016; Langer et al. 2017), or whether more quasi-static cloud contraction processes take place where ever increasing densities may be related to the phase

^ Data are only available at the CDS via anonymous ftp to cdsarc.u-strasbg.fr $(130.79 .128 .5)$ or via http://cdsarc. u-strasbg.fr/viz-bin/cat/J/A+A/638/A44 transitions between the atomic and molecular gas (e.g., Elmegreen 1993; Williams et al. 2000; McKee \& Ostriker 2007).

In dynamical pictures of converging gas flows, cloud-cloud collisions and cloud collapse flows, a convergence of gas flows toward some point in space is involved. There is a major difference between converging gas flows and cloud-cloud collisions on the one hand and cloud collapse on the other: in the converging gas flow and cloud-cloud collision picture the compression is produced by some external cause (e.g., supernovae or spiral arm potentials, e.g., Mac Low \& Klessen 2004; Haworth et al. 2018; Kobayashi et al. 2018), whereas the collapsing cloud flows are dominated by the self-gravity of the cloud itself (e.g., VázquezSemadeni et al. 2019). In reality, externally driven converging flows may produce the clouds that then further collapse under their own self-gravity.

The observational characterization of the transition from atomic to molecular gas requires observations of both phases in atomic and molecular spectral lines, respectively. Most molecular cloud studies have been conducted in different lines of carbon monoxide covering a large range of spatial scales (e.g., Dame et al. 2001; Jackson et al. 2006; Dempsey et al. 2013; Barnes et al. 2015; Heyer \& Dame 2015; Rigby et al. 2016; Umemoto et al. 2017; Schuller et al. 2017). Similarly, the HI $21 \mathrm{~cm}$ line has been 


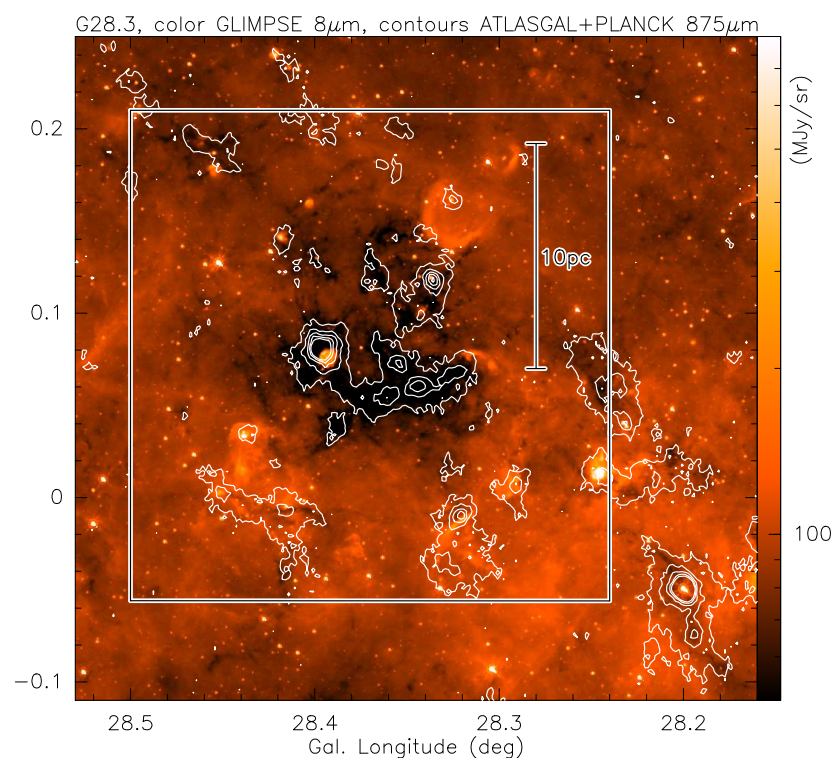

Fig. 1. Overview of the target region G28.3. The color scale presents the $8 \mu \mathrm{m}$ emission from GLIMPSE (Churchwell et al. 2009), and the contours show the $875 \mu \mathrm{m}$ emission from the ATLASGAL survey (Schuller et al. 2009, contour levels start at the $4 \sigma$ level of $200 \mathrm{mJy}^{-1}$ beam $^{-1}$ and continue in $8 \sigma$ steps up to $1.8 \mathrm{Jy} \mathrm{beam}^{-1}$ ). The white box outlines the approximate region imaged with APEX in the $[\mathrm{CI}]$ and ${ }^{13} \mathrm{CO}(3-2)$ emission. A scale bar is shown within the box.

observed often since its first detection (Ewen \& Purcell 1951); notable surveys of the Milky Way are Kalberla et al. (2005), Stil et al. (2006), McClure-Griffiths et al. (2009), Kerp et al. (2011), Winkel et al. (2016), HI4PI Collaboration (2016), Beuther et al. (2016), and Wang et al. (2020a). However, kinematic studies of the HI emission are difficult because the spectra typically cover velocity ranges of $100 \mathrm{~km} \mathrm{~s}^{-1}$ and more. With such broad line emission that traces a mixture of the cold neutral medium (CNM) and the warm neutral medium (WNM), unambiguous identification of velocity structure belonging to individual clouds is challenging (e.g., Kalberla \& Kerp 2009; Winkel et al. 2016; Beuther et al. 2016; Murray et al. 2018; Wang et al. 2020a). A different approach for studying the atomic hydrogen kinematics is to look for HI self-absorption (HISA) features at comparably high HI column densities against HI background emission. Such HISA features allow us to isolate the CNM from the WNM. Some example studies in that direction were conducted by, among others, Gibson et al. (2000, 2005a,b), Li \& Goldsmith (2003), Krčo et al. (2008), Heiner et al. (2015), and Wang et al. (2020b). In this work, we employ the HI/OH/Recombination line survey of the Milky Way (THOR, Beuther et al. 2016; Wang et al. 2020a) to study the HI kinematics from HISA features around the famous infrared dark cloud (IRDC) G28.3 (Fig. 1).

Neither molecular emission nor atomic HI emission traces the transition phase between the two media well. While the ionized carbon [CII] may trace that transition, [CII] emission depends on the local radiation field strength. In infrared dark clouds like the target cloud of this study, the [CII] emission is often too faint to be clearly detectable (e.g., Beuther et al. 2014; Clark et al. 2019). Furthermore, since [CII] cannot be observed from the ground, conducting large and sensitive maps are often difficult to obtain. Arguably, one of the best tracers of the cloud formation and transition phase is the atomic carbon fine structure line [CI] around $492 \mathrm{GHz}$ (e.g., Papadopoulos et al. 2004; Offner et al. 2014; Glover et al. 2015). The [CI] emission is believed to trace both the molecular clouds and also the more diffuse emission from the CO-dark molecular gas as well as the atomic envelope around the molecular clouds (e.g., Störzer et al. 1997; Papadopoulos et al. 2004; Offner et al. 2014; Glover et al. 2015).

Previous atomic carbon fine structure line studies typically covered only comparably small areas not extending far into the more diffuse cloud envelope structures (e.g., Keene 1995; Schilke et al. 1995; Ossenkopf et al. 2011). In an attempt to study the early evolutionary stages of high-mass star formation, we investigated four IRDCs in ionized, atomic, and molecular carbon (Beuther et al. 2014). In one of the clouds (G48.66) we found evidence of converging gas flows in [CII] emission. However, even in that case the size of the maps was comparably small, and did not reach far into the environmental cloud.

Here we are presenting an atomic carbon [CI] study of the prototypical IRDC G28.3 at larger scales of roughly $15^{\prime} \times 15^{\prime}$ or $\sim 20 \times 20 \mathrm{pc}^{2}$. The molecular cloud G28.3 is a large region where the innermost region is the well-known IRDC G28.3 (e.g., Pillai et al. 2006; Wang et al. 2008; Ragan et al. 2012; Butler \& Tan 2012; Butler et al. 2014; Tan et al. 2013; Tackenberg et al. 2014; Kainulainen \& Tan 2013; Zhang et al. 2015; Feng et al. 2016a). Infrared data from the Spitzer satellite show that filamentary extinction structures extend from the large-scale atomic and molecular outskirts down to the innermost cloud center (Fig. 1, Churchwell et al. 2009). At a kinematic distance of $4.7 \mathrm{kpc}$, observations of the dense gas in $\mathrm{N}_{2} \mathrm{H}^{+}$indicate that the region is in a stage of global collapse (Tackenberg et al. 2014), and different signs of star formation activity exist throughout the cloud (e.g., Wang et al. 2008; Butler \& Tan 2012; Tan et al. 2016; Feng et al. 2016b). The $\mathrm{N}_{2} \mathrm{H}^{+}(1-0)$ data of the central regions indicate a velocity spread with peak velocities varying approximately between 77.5 and $81.5 \mathrm{~km} \mathrm{~s}^{-1}$ (Tackenberg et al. 2014). In the following we use an approximate velocity of rest of $v_{\mathrm{lsr}} \sim 79.5 \mathrm{~km} \mathrm{~s}^{-1}$. The above characteristics make the G28.3 complex an ideal candidate to investigate the cloud formation and atomic to molecular gas conversion processes.

In the following, we combine an analysis of the diffuse environmental atomic and molecular cloud traced by HISA and ${ }^{13} \mathrm{CO}(1-0)$ emission to the denser G28.3 IRDC center better studied in the $[\mathrm{CI}]$ and ${ }^{13} \mathrm{CO}(3-2)$ transitions.

\section{Observations and data}

\subsection{THOR $\mathrm{HI}$ and ${ }^{13} \mathrm{CO}(1-0) \mathrm{GRS}$ data}

The HI data are taken from the THOR survey of the Milky Way (Beuther et al. 2016; Wang et al. 2020a) conducted with the Very Large Array (VLA). The final atomic hydrogen HI data product is the combined data cube from the THOR C-array observations with the previous VLA D-array survey and GBT single-dish data (VGPS, Stil et al. 2006). The angular and spectral resolution and the typical rms of the combined dataset are $40^{\prime \prime}, 1.5 \mathrm{~km} \mathrm{~s}^{-1}$, and $10 \mathrm{mJy}$ beam $^{-1}$, respectively. For more details about the survey and data products, see Beuther et al. (2016) and Wang et al. $(2020 a)^{1}$.

The corresponding large-scale ${ }^{13} \mathrm{CO}(1-0)$ data are taken from the Galactic Ring Survey GRS (Jackson et al. 2006). The spatial and spectral resolution as well as the typical rms sensitivity of this survey are $46^{\prime \prime}, 0.21 \mathrm{~km} \mathrm{~s}^{-1}$, and $0.13 \mathrm{~K}$, respectively.

http://www .mpia.de/thor 


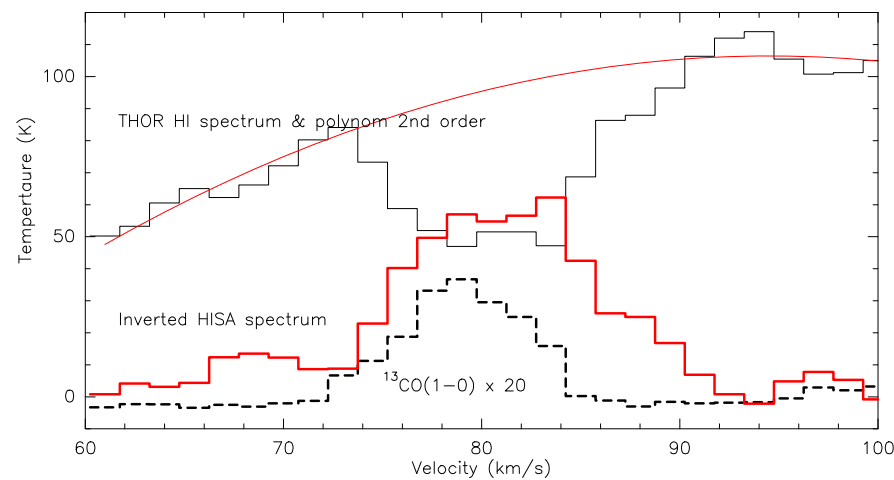

Fig. 2. Compilation of $\mathrm{HI}$ and ${ }^{13} \mathrm{CO}(1-0)$ spectra for the central area of the G28.3 IRDC (averaged over the central 100" squared). The thin black histogram shows the original $\mathrm{HI}$ emission data from the THOR survey (Beuther et al. 2016; Wang et al. 2020a) where the HI selfabsorption (HISA) is visible as absorption against the typically bright $\sim 100 \mathrm{~K}$ emission of the Galaxy. The red line presents a second-order polynomial fit to the non-HISA part of the spectrum. The red histogram then shows the resulting inverted HISA spectrum for this feature. The dashed histogram presents the ${ }^{13} \mathrm{CO}(1-0)$ emission (Jackson et al. 2006) for the same region (multiplied by 20 for easier readability).

\section{2. $\mathrm{APEX}[\mathrm{Cl}]$ and ${ }^{13} \mathrm{CO}(3-2)$ observations}

The atomic carbon $[\mathrm{CI}]$ and ${ }^{13} \mathrm{CO}(3-2)$ data were observed with the APEX telescope ${ }^{2}$ in several observing runs between May and July 2018 (project ID M9504A_101). The approximate area of $0.25 \times 0.25$ covered in the on-the-fly mode is outlined by the white box in Figs. 1, 3, and 4. The two-band FLASH receiver was tuned to the [CI] frequency of $492.160651 \mathrm{GHz}$ and to the ${ }^{13} \mathrm{CO}(3-2)$ frequency of $330.587965 \mathrm{GHz}$. Maps were conducted in the on-the-fly mode, always with maps in right ascension and declination to reduce potential scanning effects. The data reduction was conducted within the GILDAS framework with the subprograms class and greg ${ }^{3}$.

The original spectral resolution is $\sim 0.05 \mathrm{~km} \mathrm{~s}^{-1}$. However, to decrease the noise, we binned the data to a spectral resolution of $0.5 \mathrm{~km} \mathrm{~s}^{-1}$. To increase the signal-to-noise ratio for the [CI] data, we smoothed them to a spatial resolution of $20^{\prime \prime}$. The ${ }^{13} \mathrm{CO}(3-2)$ data are kept at their native spatial resolution of $18^{\prime \prime}$. The data are in antenna temperature $T_{\mathrm{A}}^{*}$. The rms for the $[\mathrm{CI}]$ and ${ }^{13} \mathrm{CO}(3-2)$ data cubes in a single $0.5 \mathrm{~km} \mathrm{~s}^{-1}$ channel is 0.14 and $0.12 \mathrm{~K}$, respectively.

\section{Results}

\subsection{Large-scale atomic $\mathrm{HI}$ and molecular gas ${ }^{13} \mathrm{CO}(1-0)$ distribution}

The THOR atomic hydrogen data reveal a clear HI selfabsorption (HISA) cloud in the environment of G28.3 (Figs. 2 and 3). While such a dip in HI emission could also be caused by missing $\mathrm{HI}$, the fact that this $\mathrm{HI}$ emission dip at the given velocities is correlated with strong ${ }^{13} \mathrm{CO}$ emission indicates that the lower HI emission is most likely caused by HI selfabsorption (e.g., Riegel \& Crutcher 1972; Heiles \& Gordon 1975; van der Werf et al. 1988; Gibson et al. 2000; Kavars et al. 2005; Dénes et al. 2018; Wang et al. 2020b). Furthermore, we checked

2 APEX, the Atacama Pathfinder Experiment is a collaboration between the Max-Planck-Institut für Radioastronomie, the Onsala Space Observatory (OSO), and the European Southern Observatory (ESO).

3 For more details see http://wWw.iram. fr/IRAMFR/GILDAS
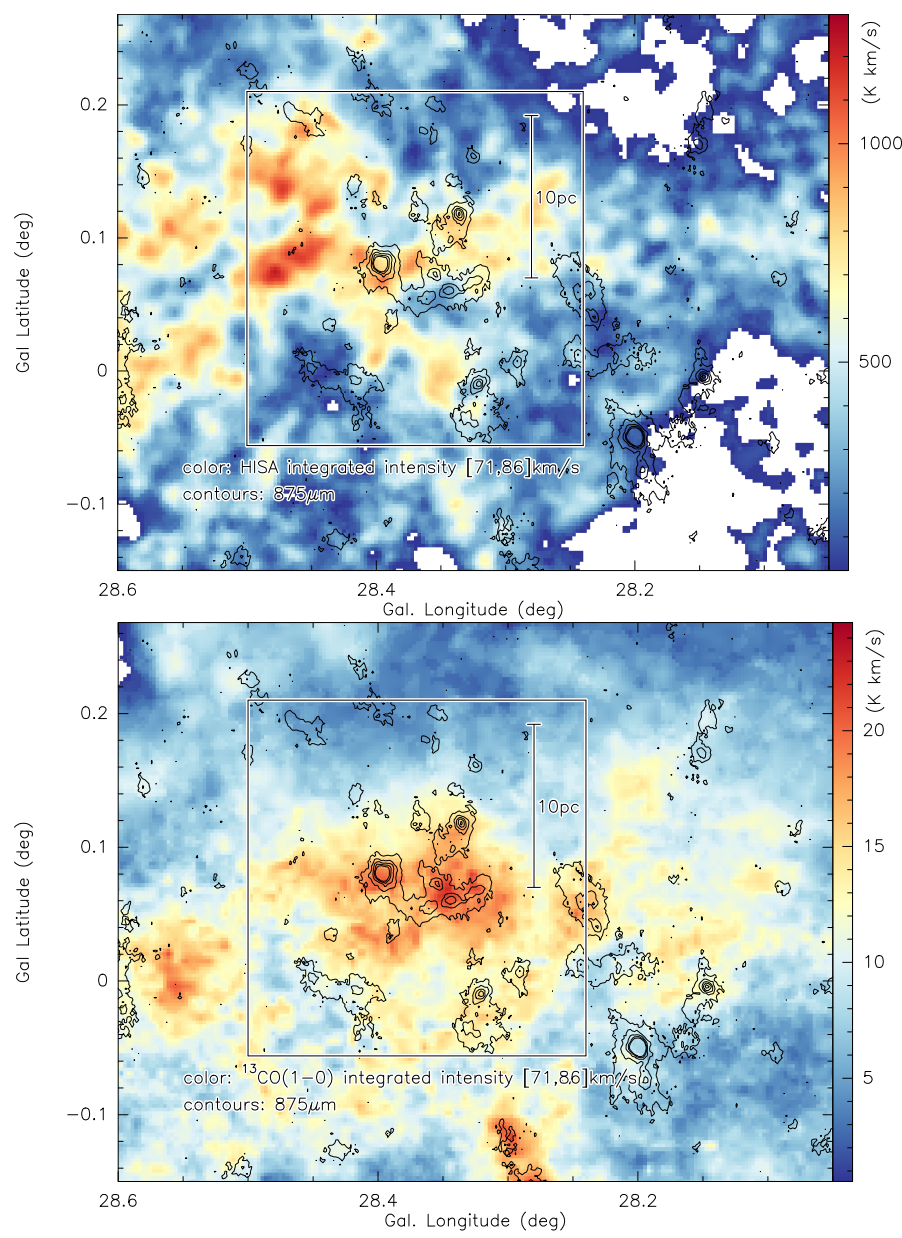

Fig. 3. Large-scale structure of the cold neutral medium (CNM) measured as HISA in the THOR survey (top panel, Beuther et al. 2016; Wang et al. 2020a,b) and the ${ }^{13} \mathrm{CO}(1-0)$ emission from GRS (bottom panel, Jackson et al. 2006). The color scales show the inverted HISA emission and ${ }^{13} \mathrm{CO}(1-0)$ maps, respectively (integrated from 71 to $86 \mathrm{~km} \mathrm{~s}^{-1}$ above $15 \mathrm{~K}$ in the HISA spectrum), and the contours show the $875 \mu \mathrm{m}$ emission from the ATLASGAL survey (Schuller et al. 2009, contour levels start at the $4 \sigma$ level of $200 \mathrm{mJy} \mathrm{beam}^{-1}$ and continue in $8 \sigma$ steps up to $1.8 \mathrm{Jy} \mathrm{beam}^{-1}$ ). The white box outlines the approximate region imaged with APEX in the $[\mathrm{CI}]$ and ${ }^{13} \mathrm{CO}(3-2)$ emission. A scale bar is shown within the box.

the THOR centimeter continuum data for background sources against which we can directly measure the absorption. Although we have only weak continuum sources in that field prohibiting indepth real absorption studies, we do find HI absorption against the continuum at the respective velocity ranges. This confirms high HI column densities and hence the HISA interpretation in general for the G28.3 cloud.

Since HISA features are absorption signatures against the bright HI emission of the Milky Way, they trace the CNM (e.g., Li \& Goldsmith 2003; Gibson et al. 2000, 2005a,b; Wang et al. 2020b). Fitting a second-order polynomial to the channels around the HISA feature, and inverting the resulting spectra, it is possible to retrieve a HISA spectrum (see Fig. 2 showing the different parts of that approach). For more details about the actual HISA extraction process, see Wang et al. (2020b).

In comparison to the normal HI spectrum with emission at almost all velocities, this HISA spectrum has the advantage that it is almost Gaussian and allows us to perform a much simpler analysis of the CNM than any normal HI emission spectra would 


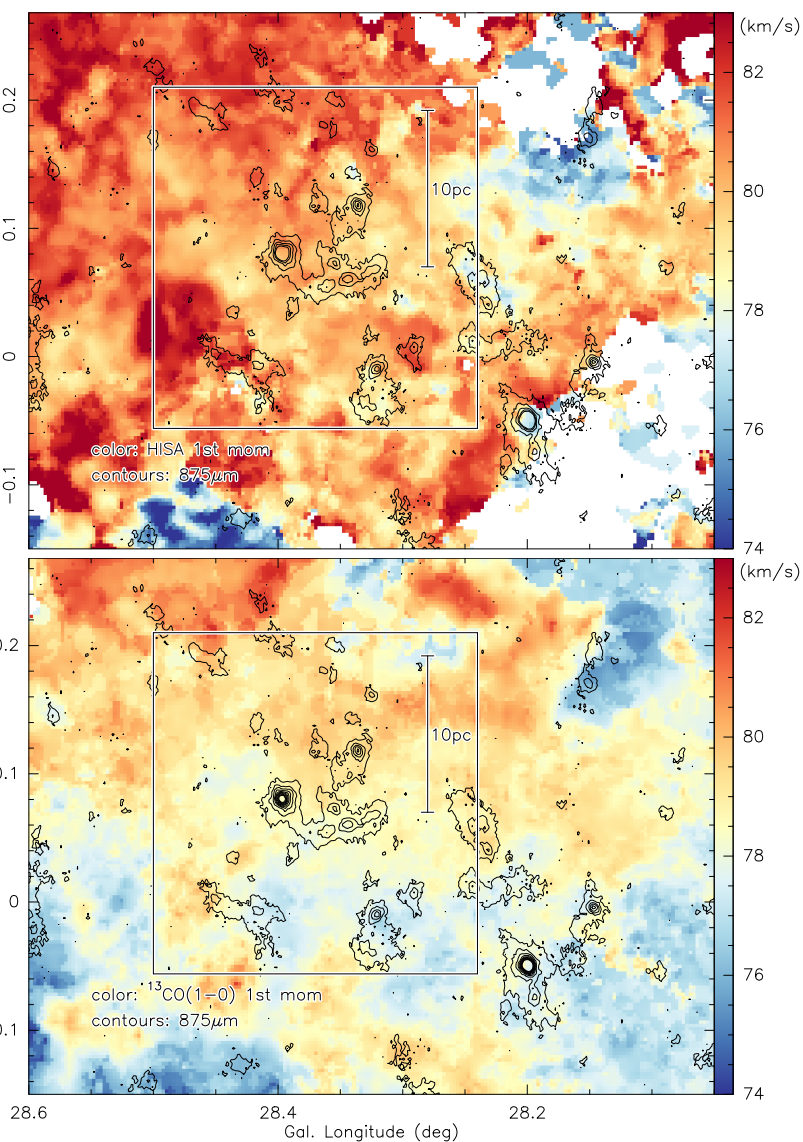

Fig. 4. Large-scale velocity structure of the cold neutral medium (CNM) measured as HISA (top panel) and of the molecular gas measured in ${ }^{13} \mathrm{CO}(1-0)$ (bottom panel, Jackson et al. 2006). The color scale shows in both panels the first moment maps (intensity-weighted peak velocities), and the contours show the $875 \mu \mathrm{m}$ emission from the ATLASGAL survey (Schuller et al. 2009; contour levels start at the $4 \sigma$ level of $200 \mathrm{mJy}$ beam $^{-1}$ and continue in $8 \sigma$ steps up to $1.8 \mathrm{Jy} \mathrm{beam}^{-1}$ ). The white box outlines the approximate region imaged with APEX in the $[\mathrm{CI}]$ and ${ }^{13} \mathrm{CO}(3-2)$ emission. Scale bars are shown within the box.

do. Doing this HISA fitting pixel by pixel, we can derive a HISA emission map. Figure 3 presents this HISA map integrated over the velocity of the cloud between 71 and $86 \mathrm{~km} \mathrm{~s}^{-1}$, and clearly shows that the general structure of the CNM traced by the HISA is associated with the infrared dark cloud G28.3, but that the CNM appears as expected to be more extended. While the central $875 \mu \mathrm{m}$ emission appears to be embedded in a larger scale envelope of CNM, the HISA peak emission is offset from the $875 \mu \mathrm{m}$ main filamentary structure. This decrease in HISA toward the main infrared dark filament may be indicative of ongoing conversion of atomic to molecular gas in the central denser molecular cloud, also traced by the $875 \mu \mathrm{m}$ emission.

Furthermore, the HISA data cube allows us to extract moment maps to study the velocity structure, which is similar to typical approaches conducted with molecular line data. Figure 4 presents the corresponding first moment maps (intensity-weighted peak velocities) of the HISA as well as the ${ }^{13} \mathrm{CO}(1-0)$ data from GRS (Jackson et al. 2006). The general gas velocities of the CNM traced by the HISA and the molecular gas traced by the ${ }^{13} \mathrm{CO}(1-0)$ emission cover the same velocities between approximately 70 and $90 \mathrm{~km} \mathrm{~s}$ (Figs. 2 and 4). Hence, both should trace approximately the same large-scale structures in our Milky Way. However, there are also significant kinematic differences. While the molecular gas shows a velocity gradient across Galactic latitudes (similar to the finding of the even denser gas seen in $\mathrm{N}_{2} \mathrm{H}^{+}$on smaller scales by Tackenberg et al. 2014) from $\sim 83$ to $\sim 76 \mathrm{~km} \mathrm{~s}^{-1}$, the HISA first moment map instead shows more uniform velocities around $82-83 \mathrm{~km} \mathrm{~s}^{-1}$ in the outskirts of the cloud with slightly lower velocities toward the center of the G28.3 region. While this slight shift may also be indicative of a small gradient in the HISA map, this is observationally not significant (see also position-velocity discussion in Sect. 3.2). We return to the velocity gradients in comparison to the [CI] emission in the following section.

\subsection{Velocity structure of atomic and molecular carbon around the G28.3 IRDC}

In the following, we focus on the closer environment around the IRDC G28.3. Figure 5 presents the integrated [CI] and ${ }^{13} \mathrm{CO}(3-2)$ emission from our APEX observations. While both tracers show the strongest emission toward the $875 \mu \mathrm{m}$ peak positions, the ${ }^{13} \mathrm{CO}(3-2)$ emission follows the dense gas structures more closely than the atomic carbon $[\mathrm{CI}]$ emission, which exhibits a more diffuse halo-like structure around the central $875 \mu \mathrm{m}$ dust continuum emission.

More interesting than just the integrated emission are the kinematic signatures found in both tracers. Figure 6 shows the first moment maps in [CI] and ${ }^{13} \mathrm{CO}(3-2)$. A clear velocity gradient can be seen in both tracers from roughly $83 \mathrm{~km} \mathrm{~s}^{-1}$ at latitudes higher than the central IRDC, and velocities even below $74 \mathrm{~km} \mathrm{~s}^{-1}$ at latitudes below the IRDC. The main dust continuum filament around a latitude of $\sim 0.07$ exhibits a rather uniform peak velocity around $\sim 78 \mathrm{~km} \mathrm{~s}^{-1}$, whereas the additional strong $875 \mu \mathrm{m}$ peak at higher latitudes $(\sim 0.12)$ is already more redshifted with velocities $>80 \mathrm{~km} \mathrm{~s}^{-1}$. This velocity shift was already identified in the high-density tracer $\mathrm{N}_{2} \mathrm{H}^{+}$by Tackenberg et al. (2014).

Particularly prominent is a velocity feature at Galactic coordinates of $\sim 28^{\circ} .33 / 0^{\circ} .01$ where the first moment maps in Fig. 6 show a strong decrease to even lower velocities, and at the same position the second moment maps exhibit a jump to low velocitydispersion measurements (see black rectangles in Figs. 6 and 7). Since moment maps are only intensity-weighted integral measurements, to investigate this velocity change in more depth, we extracted individual [CI] and ${ }^{13} \mathrm{CO}(3-2)$ spectra toward four positions along that velocity structure labeled 1-4 in Figs. 6 and 7. These spectra are shown in Fig. 8.

The $[\mathrm{CI}]$ and ${ }^{13} \mathrm{CO}(3-2)$ spectra toward that region clearly exhibit several velocity components. While the southernmost position 1 shows mainly one velocity component around 73$74 \mathrm{~km} \mathrm{~s}^{-1}$, the northernmost position 4 is dominated by a (broader) component at around $79 \mathrm{~km} \mathrm{~s}^{-1}$. The two positions in between, and particularly prominent toward position 3 show two-component spectra combining the features seen individually toward positions 1 and 4 .

Having two different velocity components in the environment of that IRDC raises the question of whether we are witnessing the potential interaction of two gas flows. These could be either externally driven colliding flows and/or cloud-cloud collisions, or they could be driven by self-gravity, which may trigger the formation of a dense star-forming molecular cloud at its converging point (e.g., Duarte-Cabral et al. 2011; Bisbas et al. 2017; Inoue et al. 2018; Kobayashi et al. 2018; Haworth et al. 2015, 2018; Vázquez-Semadeni et al. 2006, 2019; Heitsch et al. 2008; Banerjee et al. 2009; Gómez \& Vázquez-Semadeni 2014). Regarding the externally driven processes, we can consider the 


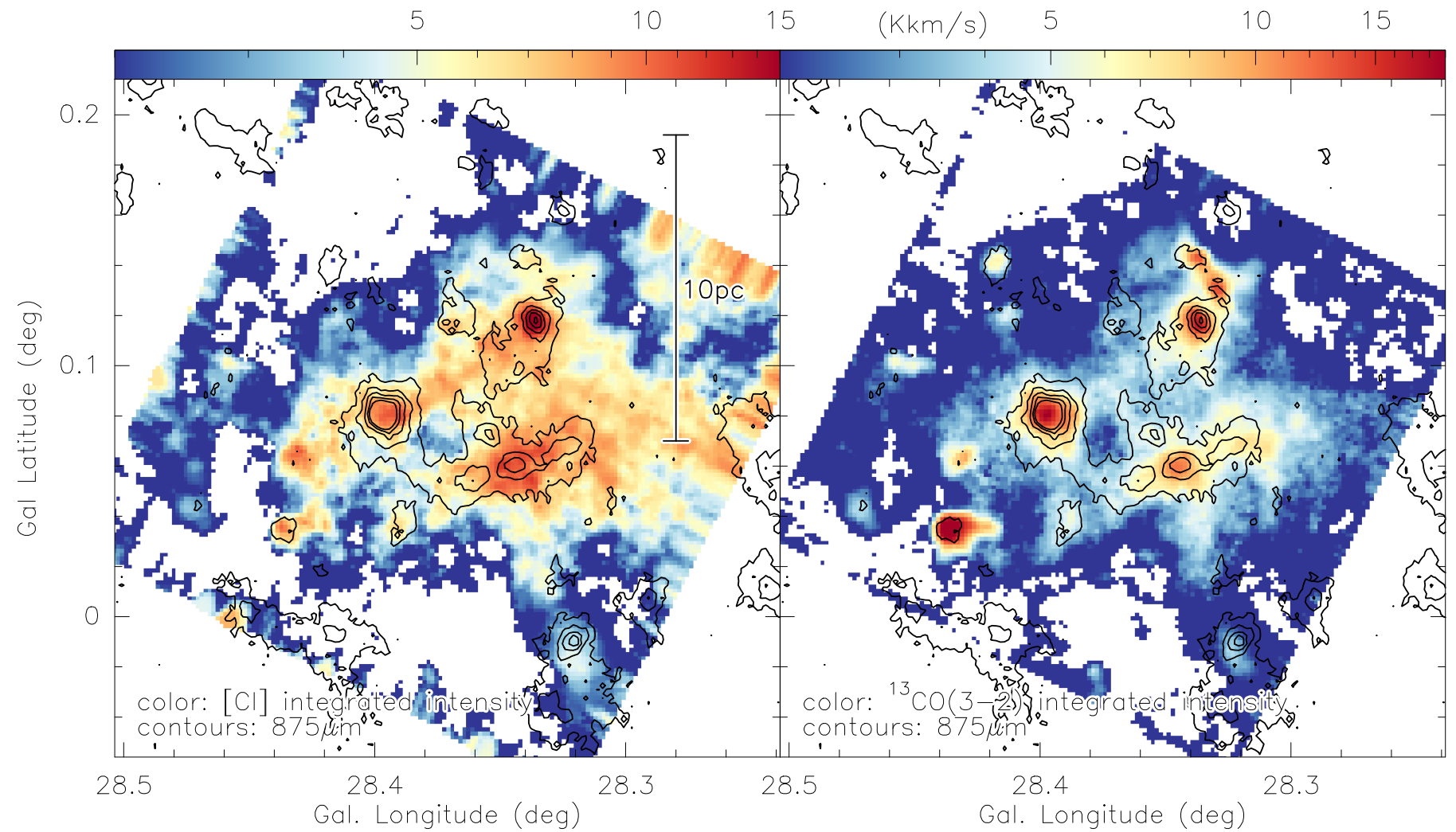

Fig. 5. Integrated intensity maps of $[\mathrm{CI}]$ (left panel) and ${ }^{13} \mathrm{CO}(3-2)$ (right panel). The color scales present the corresponding integrated line maps over a velocity range of $[71,86] \mathrm{km} \mathrm{s}^{-1}$. The contours show the $875 \mu \mathrm{m}$ emission from the ATLASGAL survey (Schuller et al. 2009, contour levels start at the $4 \sigma$ level of $200 \mathrm{mJy}_{\text {beam }}^{-1}$ and continue in $8 \sigma$ steps up to $1.8 \mathrm{Jy}_{\text {beam }}^{-1}$ ). A scale bar is shown in the left panel.

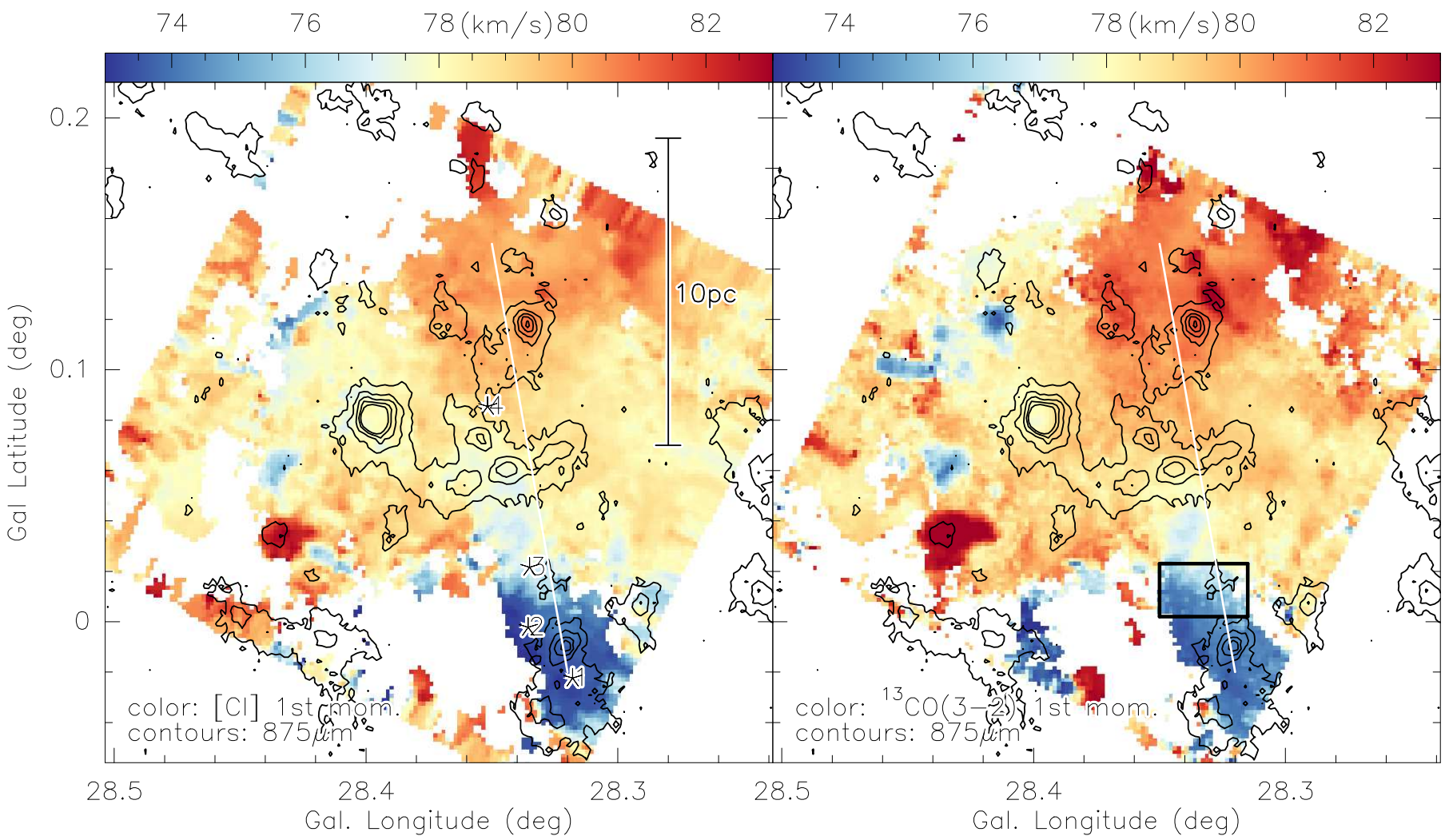

Fig. 6. First moment maps (intensity-weighted peak velocities) of [CI] (left panel) and ${ }^{13} \mathrm{CO}(3-2)$ (right panel) over a velocity range of $[71,86] \mathrm{km} \mathrm{s}^{-1}$. The contours show the $875 \mu \mathrm{m}$ emission from the ATLASGAL survey (Schuller et al. 2009; contour levels start at the $4 \sigma$ level of $200 \mathrm{mJy} \mathrm{beam}^{-1}$ and continue in $8 \sigma$ steps up to $1.8 \mathrm{Jy} \mathrm{beam}^{-1}$ ). The white lines show the direction (north to south) of the pv-diagrams in Fig. 9 . The stars in the left panel show the positions of the spectra presented in Fig. 8. A scale bar is shown in the left panel. The black rectangle in the right panel outlines the region with the velocity jump. 


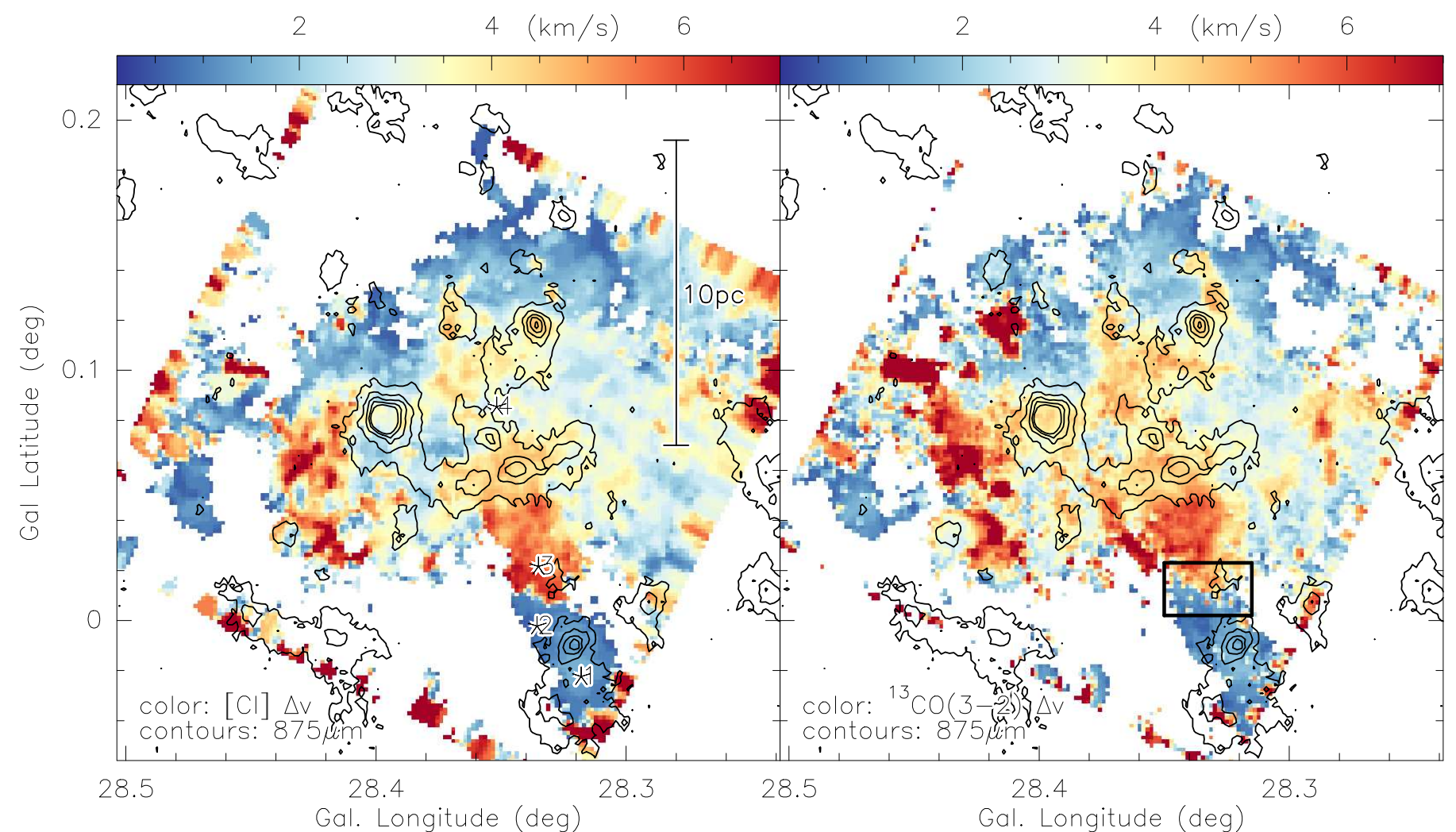

Fig. 7. Second moment maps (intensity-weighted velocity dispersions) of [CI] (left panel) and ${ }^{13} \mathrm{CO}(3-2)$ (right panel) over a velocity range of $[71,86] \mathrm{km} \mathrm{s}^{-1}$. The contours show the $875 \mu \mathrm{m}$ emission from the ATLASGAL survey (Schuller et al. 2009; contour levels start at the $4 \sigma$ level of $200 \mathrm{mJy}_{\text {beam }^{-1}}$ and continue in $8 \sigma$ steps up to $1.8 \mathrm{Jy} \mathrm{beam}^{-1}$ ). The stars in the left panel show the positions of the spectra presented in Fig. 8. A scale bar is shown in the left panel. The black rectangle in the right panel outlines the region with the velocity jump.

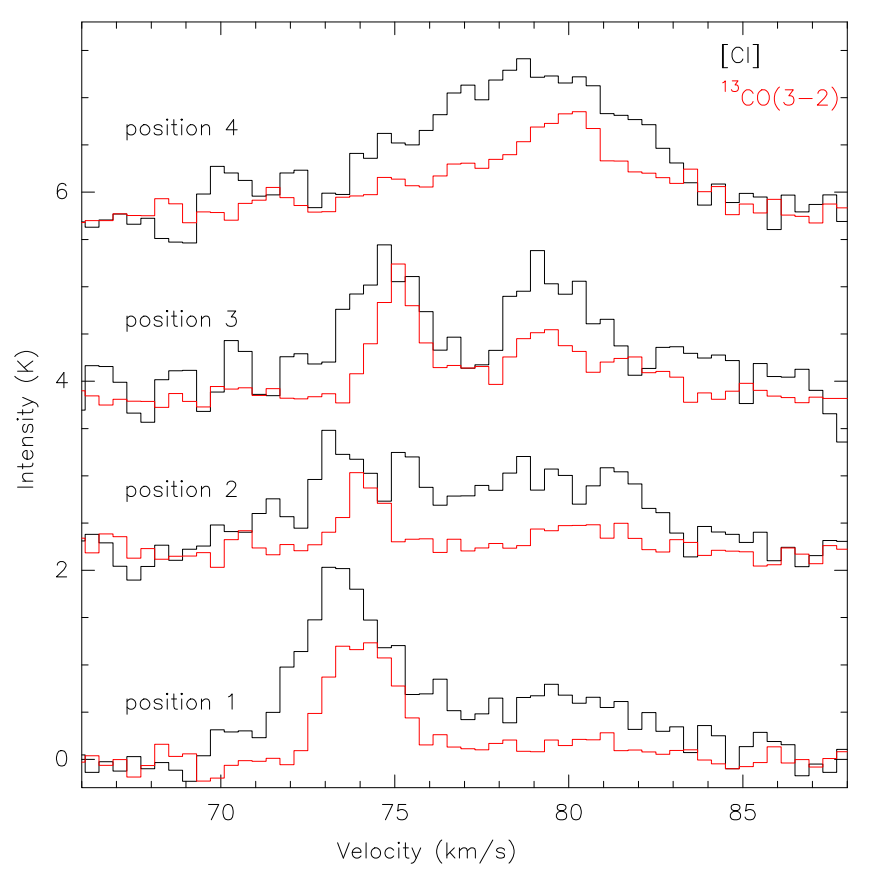

Fig. 8. [CI] (black) and ${ }^{13} \mathrm{CO}(3-2)$ (red) spectra toward the four positions indicated in Figs. 6 and 7. The vertical y-axis offsets between the spectra are only for better readability.

cloud-cloud collisions as a subgroup of the more general colliding gas flows. The main difference should be the state of the gas. While colliding flows are in general considered to be continuous gas flows that start as more diffuse HI clouds with a mix of CNM and WNM, the cloud-cloud collision picture typically refers to more concrete objects consisting mainly of cold molecular gas (e.g., Haworth et al. 2015, 2018; Bisbas et al. 2017).

To better evaluate such potential converging gas flows, Fig. 9 presents position-velocity cuts approximately along the northsouth direction in all four gas tracers, $\mathrm{HI},{ }^{13} \mathrm{CO}(1-0),[\mathrm{CI}]$, and ${ }^{13} \mathrm{CO}(3-2)$. The corresponding cut directions for the two pairs of tracers $\left(\mathrm{HI} /{ }^{13} \mathrm{CO}(1-0)\right.$ for the larger scales, and $[\mathrm{CI}] /{ }^{13} \mathrm{CO}(3-$ 2) for the closer environment of the IRDC) are shown in Figs. 10 and 6, respectively. While for the larger-scale HISA and ${ }^{13} \mathrm{CO}(1-0)$ data, we select the most straightforward north-south direction, for the $[\mathrm{CI}]$ and ${ }^{13} \mathrm{CO}(3-2)$ emission, the cut is slightly inclined $\left(\sim 10^{\circ}\right.$, Fig. 6$)$ to follow the larger extent of the $[\mathrm{CI}]$ and ${ }^{13} \mathrm{CO}(3-2)$ emission along that axis. We checked more orientations on the different data, and this small difference does not change the results discussed below. The orientation of the pvdiagrams is north-south, i.e., offset $0^{\prime \prime}$ is the northern end of the cut.

These pv-diagrams exhibit a few interesting features. To start with the diffuse emission, the HISA pv-diagram shows no obvious velocity gradient over the whole extent of $\sim 800^{\prime \prime}$ (roughly $18.25 \mathrm{pc}$ at $4.7 \mathrm{kpc}$ distance). Going to the molecular gas, the ${ }^{13} \mathrm{CO}(1-0)$ cut peaks in the north at roughly $80.5 \mathrm{~km} \mathrm{~s}^{-1}$, stays in that regime for about 300", and then exhibits a gradient toward the center of the filament to $\sim 77.5 \mathrm{~km} \mathrm{~s}^{-1}$. Of particular interest is the regime south of the main filament where the ${ }^{13} \mathrm{CO}(1-0)$ emission shows two components, one again around $80.5 \mathrm{~km} \mathrm{~s}^{-1}$ and the second component at lower velocities of $\sim 74.5 \mathrm{~km} \mathrm{~s}^{-1}$. 

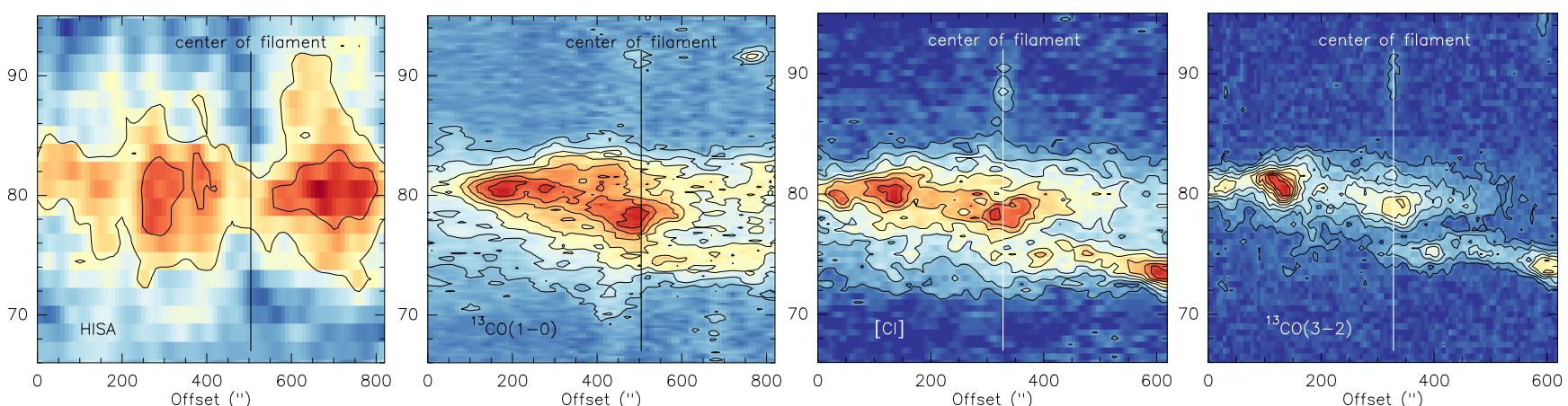

Fig. 9. Position-velocity diagrams for the four tracers from left to right: $\mathrm{HISA},{ }^{13} \mathrm{CO}(1-0),[\mathrm{CI}]$, and ${ }^{13} \mathrm{CO}(3-2)$. The cuts are in a north-south direction along the lines shown in Figs. 6 ([CI], ${ }^{13} \mathrm{CO}(3-2)$ ) and 10 (HISA, ${ }^{13} \mathrm{CO}(1-0)$ ). Both axes have a slightly larger extent for the HISA and ${ }^{13} \mathrm{CO}(1-0)$ data compared to the $[\mathrm{CI}]$ and ${ }^{13} \mathrm{CO}(3-2)$ cuts. The approximate location of the center of the filament is marked in all panels.

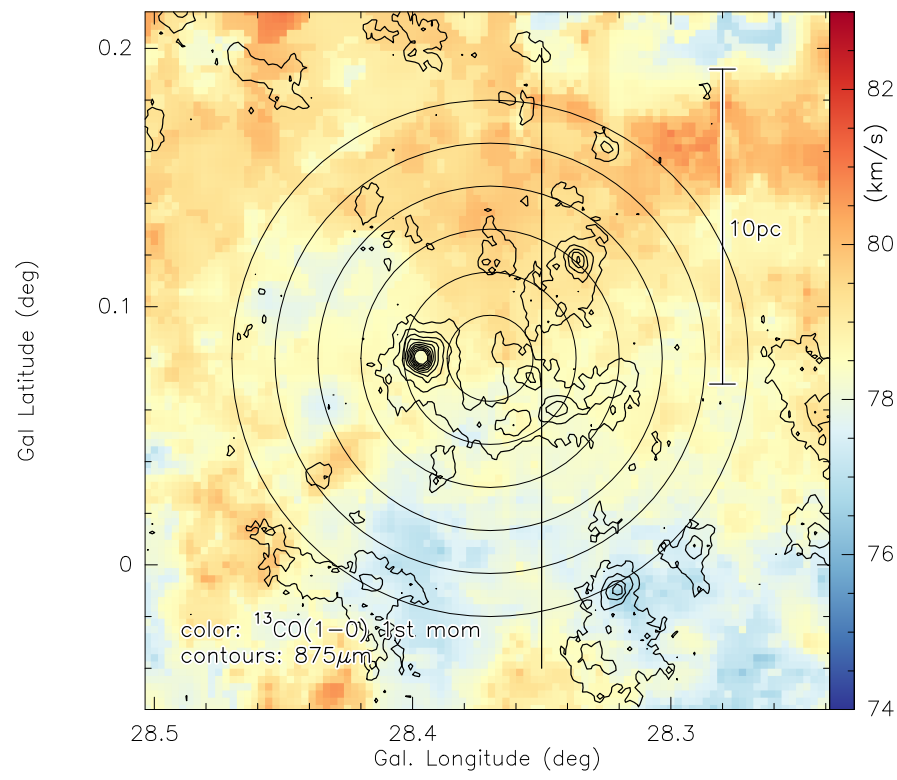

Fig. 10. Zoomed-in image of the ${ }^{13} \mathrm{CO}(1-0)$ first moment map from Fig. 4. The color scale shows the first moment map, and the contours present the ATLASGAL $875 \mu \mathrm{m}$ emission (Schuller et al. 2009; contour levels start at the $4 \sigma$ level of $200 \mathrm{mJy}_{\text {beam }}{ }^{-1}$ and continue in $8 \sigma$ steps up to $\left.1.8 \mathrm{Jy}_{\text {beam }}^{-1}\right)$. A scale bar is shown. The black line presents the direction (north to south) of the pv-diagrams in Fig. 9. The circles outline annuli with radii starting at $60^{\prime \prime}$ and then increasing in $60^{\prime \prime}$ steps to $360^{\prime \prime}$ (for details see Sect. 4.3).

The two pv-diagrams for $[\mathrm{CI}]$ and ${ }^{13} \mathrm{CO}(3-2)$ cover a slightly smaller length with about $600^{\prime \prime}$ or $\sim 13.7 \mathrm{pc}$. Around the central filament, they exhibit similar signatures with velocities around $80.5 \mathrm{~km} \mathrm{~s}^{-1}$ in the north and even below $74 \mathrm{~km} \mathrm{~s}^{-1}$ in the south. The filament itself shows again intermediate velocities between 77 and $79 \mathrm{~km} \mathrm{~s}^{-1}$. While the overall signatures for $[\mathrm{CI}]$ and ${ }^{13} \mathrm{CO}(3-2)$ are similar, $[\mathrm{CI}]$ shows a bit more extended emission with a greater velocity spread (see also Fig. 5). The division into separate velocity components is most prominent in the highest density tracer ${ }^{13} \mathrm{CO}(3-2)$. With the higher spatial and spectral resolution of the $[\mathrm{CI}]{ }^{13} \mathrm{CO}(3-2)$ data compared to the ${ }^{13} \mathrm{CO}(1-0)$ data, the gradient-like velocity structure of the two components is more clearly resolved from the north and the south toward the central filament.

For visualization purpose, Fig. 11 shows the ${ }^{13} \mathrm{CO}(3-2)$ integrated emission maps for the two velocity regimes $[71,76] \mathrm{km} \mathrm{s}^{-1}$

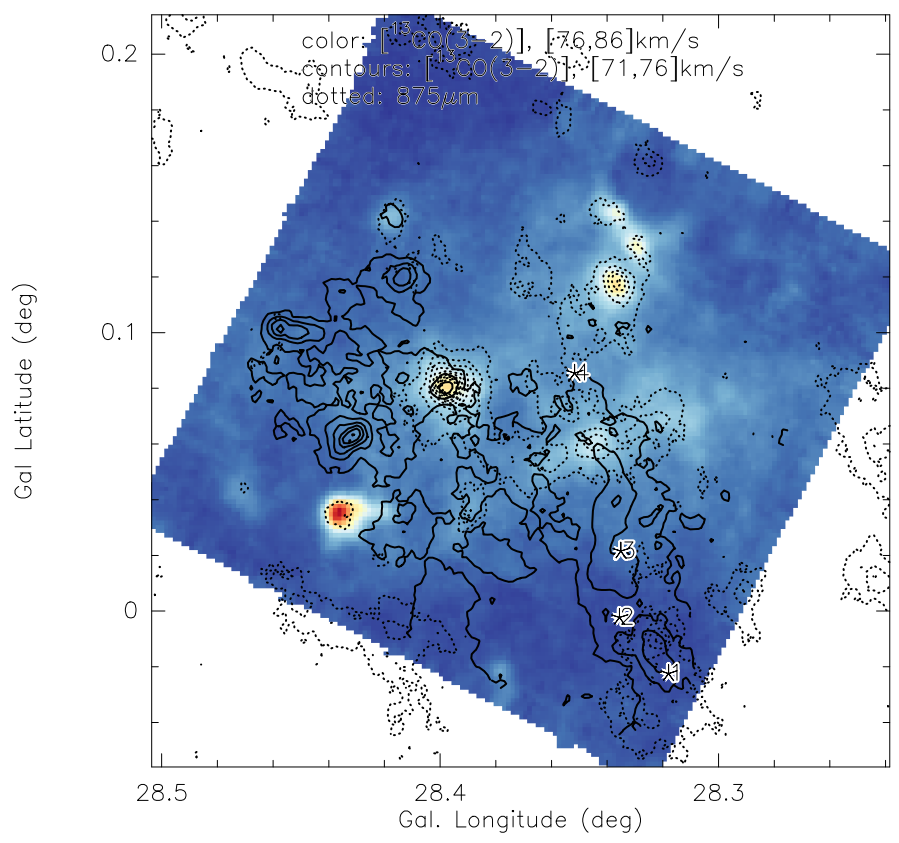

Fig. 11. Integrated ${ }^{13} \mathrm{CO}(3-2)$ emission for two velocity components. The color scale and full contours show the $[76,86]$ and $[71,76] \mathrm{km} \mathrm{s}^{-1}$ components, respectively. The contour levels are in $9 \sigma$ steps (with $\left.1 \sigma=0.125 \mathrm{~K} \mathrm{~km} \mathrm{~s}^{-1}\right)$. The dotted contours show the corresponding $875 \mu \mathrm{m}$ continuum emission from Schuller et al. (2009) as reference frame. The stars again show the positions of the spectra presented in Fig. 8.

and $[76,86] \mathrm{km} \mathrm{s}^{-1}$, respectively. Although there is no clear north-south separation of the two components, the highervelocity gas is preferentially found toward the north and the lower-velocity gas more toward the south. It should be kept in mind that these gas components and kinematic signatures are always projections onto the plane of the sky, and therefore clear spatial separations would be a surprise.

We note that while the first moment maps of ${ }^{13} \mathrm{CO}(1-0)$ and $[\mathrm{CI}] /{ }^{13} \mathrm{CO}(3-2)$ (Figs. 4 and 6) give the visual impression that the velocity gradient may increase with critical density of the tracer, this is most likely mainly caused by the two velocity components and the fact that the second component around $74.5 \mathrm{~km} \mathrm{~s}^{-1}$ is more pronounced in the higher density tracers $[\mathrm{CI}] /{ }^{13} \mathrm{CO}(3-2)$. Closer inspection of the pv-diagrams in Fig. 9 for these three tracers, considering only the transition from the north to the center of the filament, show that there is not a large 
difference in the magnitude of the velocity gradient. Similar velocity regimes were also found in the even higher-density tracer $\mathrm{N}_{2} \mathrm{H}^{+}(1-0)$ by Tackenberg et al. (2014).

Combining these different velocity signatures, the data show strong signatures of two gas components at different velocities (around $6 \mathrm{~km} \mathrm{~s}^{-1}$ apart) that converge to a common intermediate velocity at the location of the infrared dark cloud and active starforming region, similar to filament formation via gravitationally driven, converging gas flows (e.g., Gómez \& Vázquez-Semadeni 2014). We interpret these signatures as indicators of converging gas streams that may trigger the star formation event at its center (e.g., Vázquez-Semadeni et al. 2006; Heitsch et al. 2008; Banerjee et al. 2009; Gómez \& Vázquez-Semadeni 2014). Position-velocity diagrams based on simulations of cloud-cloud collisions sometimes show a characteristic pattern of lower-level emission between two main velocity components, a so-called bridging feature (e.g., Haworth et al. 2015, 2018). Similar signatures were also reported in observations (e.g., Jiménez-Serra et al. 2010; Henshaw et al. 2013; Dobashi et al. 2019; Fujita et al. 2019). The pv-diagrams of the G28.3 region presented here (Fig. 9) show different signatures in the sense that there is not a lower-intensity bridge between the two well-defined components, but that the two velocity components converge at the center of the cloud toward a central, high-intensity velocity component. However, the absences of a bridging feature does not necessarily rule out the formation of G28.3 in a cloud-cloud collision, as this feature is not always visible in simulations. For example, simulations by Bisbas et al. (2017) show that the low-intensity bridge feature may merge into a centrally peaked pv-diagram during the evolution of the cloud-cloud collisions, while the colliding-cloud simulations of Clark et al. (2019) yield only a single central velocity component in $\mathrm{CO}$ or [CI], with multiple components only becoming apparent when the [CII] emission from the cloud is considered. In addition, the multiple components and bridging feature may not be visible in cases where our line of sight is oriented at a large angle to the direction of motion of the clouds. We return to the interpretation in Sects. 4.4 and 4.5 .

\section{Discussion}

\subsection{Histogram of oriented gradients (HOG)}

A way to evaluate similarities in the structures traced by the different spectral lines is the HOG, a statistical method from machine vision recently introduced in astrophysical data analysis by Soler et al. (2019). Basically, the HOG analysis compares intensity maps by comparing the relative orientation between its gradients. The degree of correlation between the images is estimated using the projected Rayleigh statistic $(V)$, which is a test that quantifies whether the relative angle distribution is flat, as it is for two completely uncorrelated maps, or peaked around $0 \mathrm{deg}$, as it is for two maps with a significant degree of correlation.

The HOG can be used to compare individual singlefrequency maps, but also to compare position-position-velocity cubes. In that case, the result of the analysis is a matrix of $V$ values for the different velocity channels in each tracer, which is shown in Fig. 12. We use this matrix of $V$ values to investigate whether the gas traced by different spectral lines follows a similar spatial distribution across velocity channels. Soler et al. (2019) applied the HOG analysis to spectral line data as well as different simulations, and they found, for example, that constant converging gas flows result in spatial and kinematic structures agreeing well between different spectral lines (mainly showing

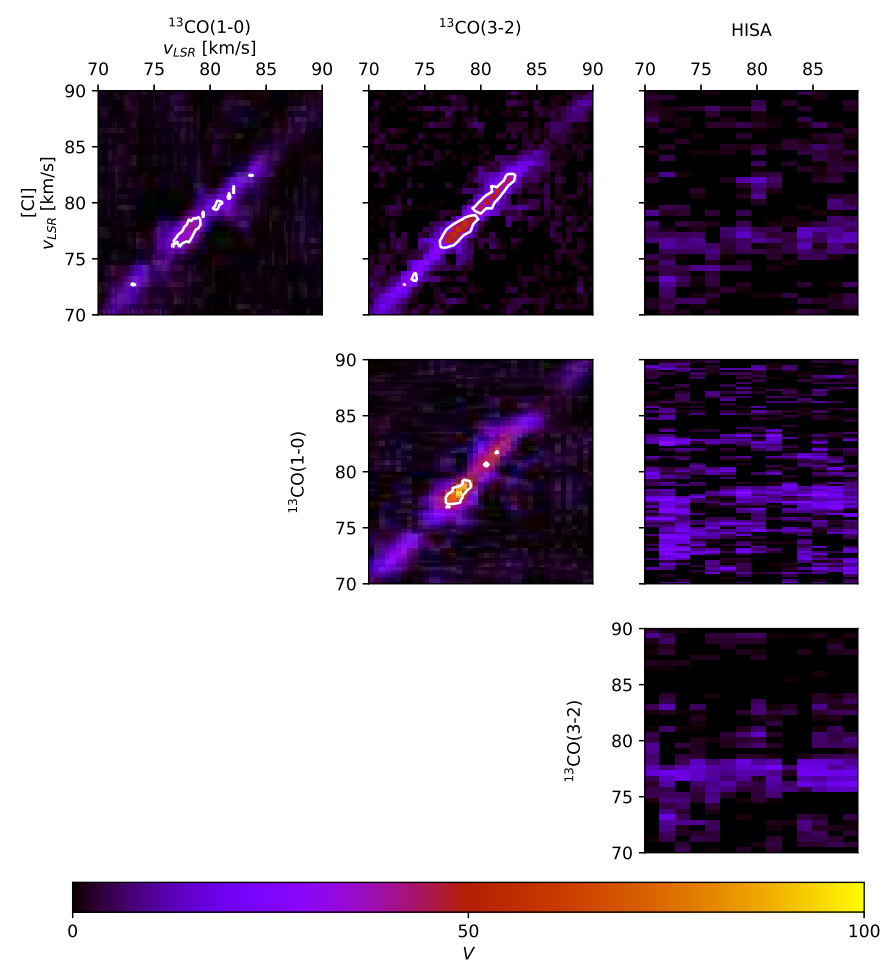

Fig. 12. Spatial correlation between the four different tracers across velocity channels as evaluated using the histogram of oriented gradients method (HOG; Soler et al. 2019). The figure shows all tracers correlated with each other as labeled at each plot. The panels show the values of the projected Rayleigh statistic $(V)$, a measure of the morphological correlation between velocity channels. A high value of $V$ indicates a strong correlation between the corresponding velocity-channel maps. The contours correspond to the $7 \sigma$ confidence limit on the $V$ values (see Soler et al. 2019 for more details).

high projected Rayleigh- $V$ values along the diagonal in plots like those in Fig. 12), whereas feedback processes can produce kinematic offsets between atomic and molecular gas (showing high projected Rayleigh- $V$ values offset from the diagonal). For more details about the method and its implications, see Soler et al. (2019).

We applied this HOG method to the four datasets studied here, namely [CI], ${ }^{13} \mathrm{CO}(1-0),{ }^{13} \mathrm{CO}(3-2)$, and HISA positionposition-velocity cubes. The resulting correlations, quantified by $V$, are presented in Fig. 12. We find that the HISA does not show significant correlation with any of the other tracers across the velocity range between 70 and $90 \mathrm{~km} \mathrm{~s}^{-1}$. In contrast, the other three tracers show high spatial correlation across velocity channels, evidenced by high $V$ values. The highest values of $V$ are concentrated along the diagonal of the correlation plot, that is, across the same line-of-sight velocity in each pair of tracers. This result is expected for tracers that are co-spatial and co-moving.

The low values of $V$ found in the comparison between the HISA and the other tracers indicate that there is no morphological correlation between CNM as traced by HISA and the other denser gas tracers across all measured velocity channels. This lack of correlation can be interpreted as an indication of the decoupling of the dense gas of the main cloud from the mostly atomic CNM in the more diffuse cloud envelope. This is slightly different to the morphological correlation found between $\mathrm{HI}$ and ${ }^{13} \mathrm{CO}(1-0)$ emission in the molecular clouds studied in Soler et al. (2019). It appears that correlation or non-correlation 
between $\mathrm{HI}$ and denser gas tracers may not be universal, but could be an indication of different evolutionary stages in this kind of objects. We note that for the G28.3 cloud studied here, the HISA itself is also weaker toward the central filamentary IRDC, as can be seen in Figs. 3 and 9. If we interprets this depression of HISA toward the center as a sign of potential gas conversion from atomic $\mathrm{H}$ to molecular $\mathrm{H}_{2}$, it can be taken as further support for the kinematic decoupling of the CNM, as traced by HISA, and the molecular phase.

\subsection{Velocity structure functions}

A different way to investigate the kinematics of molecular clouds is the analysis of velocity structure functions (e.g., Miesch \& Bally 1994; Ossenkopf \& Mac Low 2002; Esquivel \& Lazarian 2005; Heyer \& Dame 2015; Chira et al. 2019; Henshaw et al. in prep.). Velocity structure functions are essentially a measure of how much the velocity measured between pixels separated by a given distance varies as a function of spatial scale. Mathematically the structure function $S_{p}$ of order $p$ is described as

$S_{p}(l)=\left\langle|v(r)-v(r+l)|^{p}\right\rangle$,

where $l$ is known as the spatial lag and represents the separation between pairs of points where $S_{p}$ is calculated for. As outlined for example in Chira et al. (2019), the slope of the structure function can be used to infer whether turbulence and/or gravity play an important role in shaping the gas dynamics of the cloud. Chira et al. (2019) show in their simulations that purely turbulence-dominated structure functions are steeper than those where gravity becomes more important because gravity increases the velocity on small scales so that the structure function becomes shallower.

We now derived the velocity structure function $S_{2}$ of order 2 for all four gas tracers from their corresponding first moment maps (intensity-weighted peak velocities). To allow a meaningful comparison, the four first moment maps were smoothed to the $46^{\prime \prime}$ spatial resolution of the ${ }^{13} \mathrm{CO}(1-0)$ data and all put on the same pixel grid. No large-scale gradient was removed from the data. Since we derived the structure function from the first moment maps, and these are intensity-weighted peak velocities, the derived structure functions can be considered (column-)density weighted velocity structure functions.

Figure 13 presents the velocity structure function $S_{2}$ for all four tracers plotted against the spatial lag $l$. While the ${ }^{13} \mathrm{CO}(1-0)$ and $[\mathrm{CI}]$ structure functions resemble each other closely not just in the slope, but also in the magnitude of the velocity fluctuations $S_{2}$, the HISA and ${ }^{13} \mathrm{CO}(3-2)$ structure functions exhibit slightly elevated values of $S_{2}$. For ${ }^{13} \mathrm{CO}(3-2)$, this is more intuitively clear because there are very different velocities in the north and south of the region (see Figs. 6 and 9). The high absolute values of $S_{2}$ for the HISA data combined with the flat slope imply that there are comparably large velocity differences, but that these do not change strongly with spatial scale as indicated by the shallower slope.

Important information about the kinematics of the gas are encoded in the slopes of the velocity structure functions. The overall regimes of derived slopes between 0.29 and 0.68 is well within the regime of other observational studies (see, e.g., the compilation in Table 1 of Chira et al. 2019). However, while the slopes are similar for ${ }^{13} \mathrm{CO}(1-0),[\mathrm{CI}]$, and ${ }^{13} \mathrm{CO}(3-2)$ (values between 0.52 and 0.68 ), the slope of the HISA velocity structure function is flatter with a value of 0.29 .

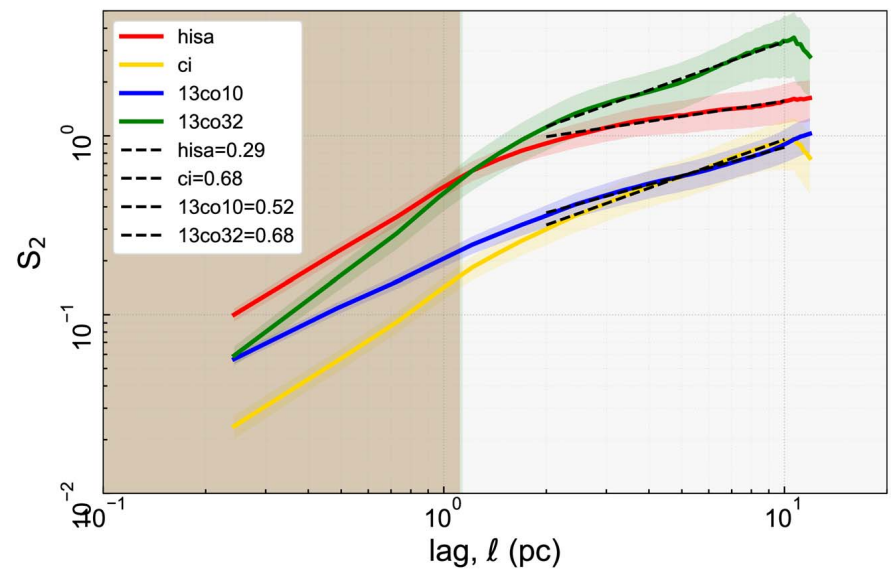

Fig. 13. One-dimensional velocity structure function. The structure function $S_{2}$ is plotted against the spatial lag $l$ for all four observed gas tracers. The red, blue, yellow, and green lines correspond to the HISA, ${ }^{13} \mathrm{CO}(1-0)$, [CI], and ${ }^{13} \mathrm{CO}(3-2)$ data, respectively. The gray-shaded area is the spatial-resolution limit corresponding to the $46^{\prime \prime}$ beam of the ${ }^{13} \mathrm{CO}(1-0)$ data. The slopes are fitted outside that limit and are shown as dashed lines. The corresponding fit results are presented in the legend.

It should be kept in mind that other effects like noise or varying optical depth can also affect the slope of the structure function (e.g., Dickman \& Kleiner 1985; Bertram et al. 2015). While optical depth should not be a big issue for the used tracers (e.g., Shimajiri et al. 2013; Riener et al. 2020), the flatter slope of the HISA structure function needs a bit more attention when considering potential noise effects. In principle, noise can introduce a flattening of a structure function; however, this is most severe on small spatial lags (see, e.g., the recent simulations in Henshaw et al. in prep.), and we only start fitting the structure function at spatial lags clearly above the spatial resolution (Fig. 13), limiting this effect already. Furthermore, the first moment maps are derived while clipping values below an rms threshold to avoid fitting the noise. Taking the HISA data, the original rms of $\sim 10 \mathrm{mJy}^{\text {beam }}{ }^{-1}$ corresponds to a brightness temperature rms of $\sim 4 \mathrm{~K}$. The velocity structure function shown in Fig. 13 is derived with all data clipped below $15 \mathrm{~K}$. To check whether the clipping affects the results, we tested the analysis with a much more conservative clipping threshold of twice the previous value (i.e., $30 \mathrm{~K}$ ). The outcome of the test shows that within the fitting errors the slope of the HISA velocity structure function stays the same. Therefore, we infer that noise is unlikely to explain the flatter slope of the HISA velocity structure function compared to the other tracers.

While the absolute values of the slopes may be affected by some of the issues discussed above, the relative difference of almost a factor of 2 in slope between the HISA and the other tracers appears to be a solid result. In the context of the HOG analysis in the previous chapter (Sect. 4.1), the slope difference between HISA and the other tracers may be considered as further support for the decoupling of the CNM, as traced by HISA, from the denser molecular gas. The flatter HISA structure function slope indicates that the magnitude of the velocity fluctuation $S_{2}$ changes less with varying length scale than is the case for the other tracers.

Comparing the slopes of observational velocity structure functions (compiled from the literature) with their simulations, Chira et al. (2019) conclude that the observed clouds are consistent with an intermediate evolutionary stage that are 
neither purely turbulence dominated (e.g., driven by supernova remnants) nor gravitationally collapsing, but where the gas flows are dominated by the formation of hierarchical structures and cores. The cloud we are observing here around the infrared dark cloud G28.3 appears to be in a similar evolutionary stage.

\subsection{Mass flows}

With the given velocity structure, we like to derive estimates for the mass flow rates of the gas. In a simple form, the mass flow rate $\dot{M}$ in units of $M_{\odot} \mathrm{yr}^{-1}$ can be approximated via

$\dot{M}=\Sigma \cdot \Delta v \cdot r\left[M_{\odot} \mathrm{yr}^{-1}\right]$,

with the column density $\Sigma$, a velocity difference $\Delta v$, and the radius $r$. Since ${ }^{13} \mathrm{CO}(1-0)$ emission is known to be either optically thin or to exhibit only comparably low optical depth (see, e.g., the detailed analysis in Riener et al. 2020), we chose the ${ }^{13} \mathrm{CO}(1-0)$ map to estimate $\Sigma$. Furthermore, ${ }^{13} \mathrm{CO}(1-0)$ peak brightness temperatures are at most $2-3 \mathrm{~K}$ in the $\mathrm{G} 28.3$ cloud. Comparing these to typical IRDC temperatures between 15 and $20 \mathrm{~K}$ (e.g., Wienen et al. 2012; Chira et al. 2013), this is additional support for the optically thin assumption. The $\mathrm{H}_{2}$ column densities $\Sigma$ were then estimated from mean ${ }^{13} \mathrm{CO}(1-0)$ integrated intensities within the annuli. We applied standard column density calculations (e.g., Rohlfs \& Wilson 2006) using a ${ }^{13} \mathrm{CO}-$ to- $\mathrm{H}_{2}$ conversion factor following Frerking et al. (1982) and a uniform excitation temperature of $15 \mathrm{~K}$. To get a global view of the mass flow, we used circular annuli with radii starting at $60^{\prime \prime}$ from the center and then increased in $60^{\prime \prime}$ steps out to $360^{\prime \prime}$ (see Fig. 10). The circularly averaged $\mathrm{H}_{2}$ column densities range between 4.3 and $6.3 \times 10^{21} \mathrm{~cm}^{-2}$.

For the velocity difference $\Delta v$ we used the mean difference of the peak velocities measured in the ${ }^{13} \mathrm{CO}(1-0)$ first moment map at the edges of the annuli in the north and south, respectively (Fig. 10). This $\Delta v$ measurement is obviously affected by line-ofsight projection and depends on the inclination of any potential gas flow. It should hence be considered as a lower limit. As radius we use the extent of the annuli of $60^{\prime \prime}$. While each of the selections can be debated, the results are just meant to give a rough estimate of the flow rates over the extent of the cloud.

Employing this approach we can estimate the mass flow rate at varying distances from the central filament. Figure 14 presents the derived $\dot{M}$ values versus the distance of the annulus from the center of the region. Being on the order of a few times $10^{-5} M_{\odot} \mathrm{yr}^{-1}$, these flow rates appear rather constant over the extent of the cloud. The absolute values should be considered lower limits because of the two-dimensional projection of the velocity gradient on the plane of the sky. Interestingly, in the gravitationally driven cloud collapse simulation by Gómez \& Vázquez-Semadeni (2014), an accretion rate onto the central filament of $\sim 150 M_{\odot}(\mathrm{Myr})^{-1}$, corresponding to $1.5 \times 10^{-4} M_{\odot} \mathrm{yr}^{-1}$, is inferred. Taken into account that our observed values should be lower limits because of projection effects, both accretion rates appear to agree within an order of magnitude. However, we note that this comparison is based on only one cloud and one simulation, so more statistical work is needed on both sides to infer tighter constraints on the mass flow rates during cloud formation in general.

These data can be interpreted as indicative of a constant mass flow from large to small spatial scales. Such constant flow rates could, for example, occur in a self-similar gravitationally dominated collapse picture (e.g., Whitworth \& Summers 1985; Li 2018).

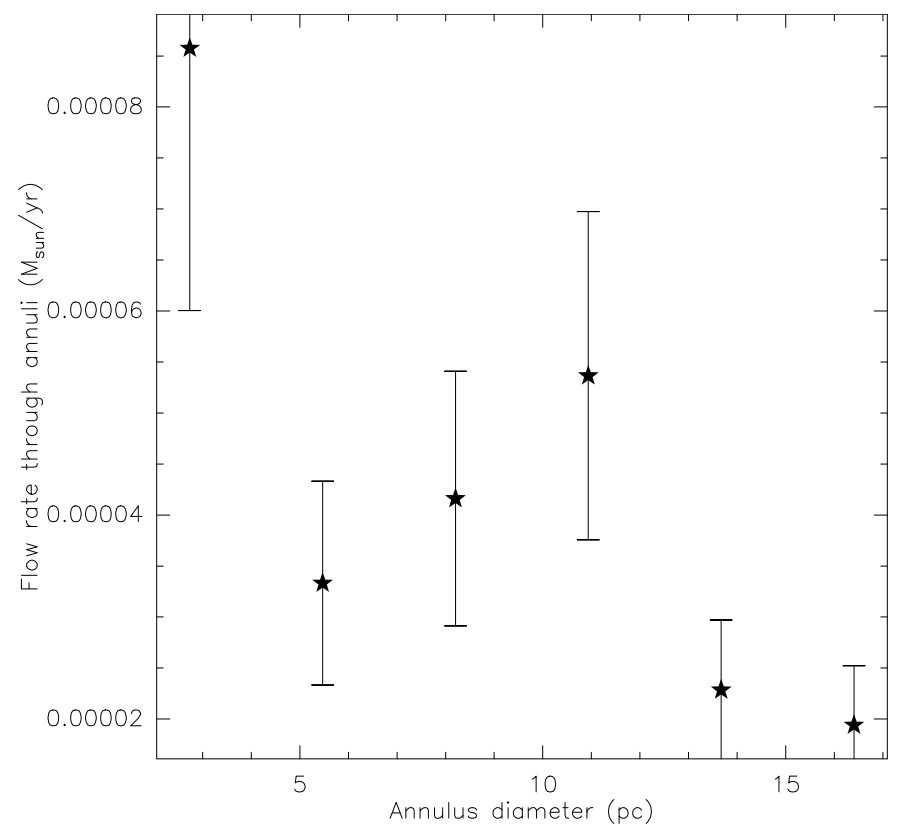

Fig. 14. Estimated mass flow rate vs. the distance of the chosen annulus from the cloud center (see Fig. 4). Error bars indicate $\pm 30 \%$ ranges.

\subsection{Comparison with simulations}

As one possible comparison of our observational results with numerical modeling studies, we choose the molecular cloud formation simulation originally presented in Gómez \& VázquezSemadeni (2014). They model the formation of the cloud of comparable size to G28.3 via the collision of two oppositely directed warm gas streams (Fig. 15). The collision triggers a phase transition to the cold medium, the cloud grows rapidly in mass until it becomes Jeans-unstable and then collapses. The successive contracting motions of the cloud are gravitationally driven. The initial low-density cloud setup of the simulation encompasses an area with diameter of $\sim 50 \mathrm{pc}$, and they form dense filaments of $\sim 15 \mathrm{pc}$ length with masses of $\sim 600 M_{\odot}$ above densities of $10^{3} \mathrm{~cm}^{-3}$. These values agree within an order of magnitude with our observed cloud and filamentary region G28.3. The collapse is simulated in the smoothed-particle hydrodynamics framework with the GADGET-2 code including self-gravity and heating and cooling functions that imply thermal bistability of the gas. For more details about the simulations, we refer to Gómez \& Vázquez-Semadeni (2014).

We focus here on their Filament 2. While the paper mainly shows the images from a snapshot at 26.6 Myr of their modeled evolution, here we present a slightly younger evolutionary stage at 21.5 Myr. At this time, the filamentary cloud is still wider and the central clump less filamentary, although it is already undergoing cloud-scale gravitational contraction, accreting from the background mostly in the direction perpendicular to the filament, and along the filament onto the central hub. This can be seen in Fig. 15 (top panel), which shows a column density projection of the cloud and velocity vectors on the plane of the figure.

Even more important are the kinematics, and Fig. 15 (bottom panel) shows a position-velocity perpendicular to the filamentary structure. In analogy to our observations, the simulation $X$-axis resembles the north-south orientation of our IRDC G28.3 data. Interestingly, at negative offsets the gas peaks at velocities of roughly $2.5 \mathrm{~km} \mathrm{~s}^{-1}$, whereas at positive offsets most of the gas is at around $-0.5 \mathrm{~km} \mathrm{~s}^{-1}$. It should be noted that at these 

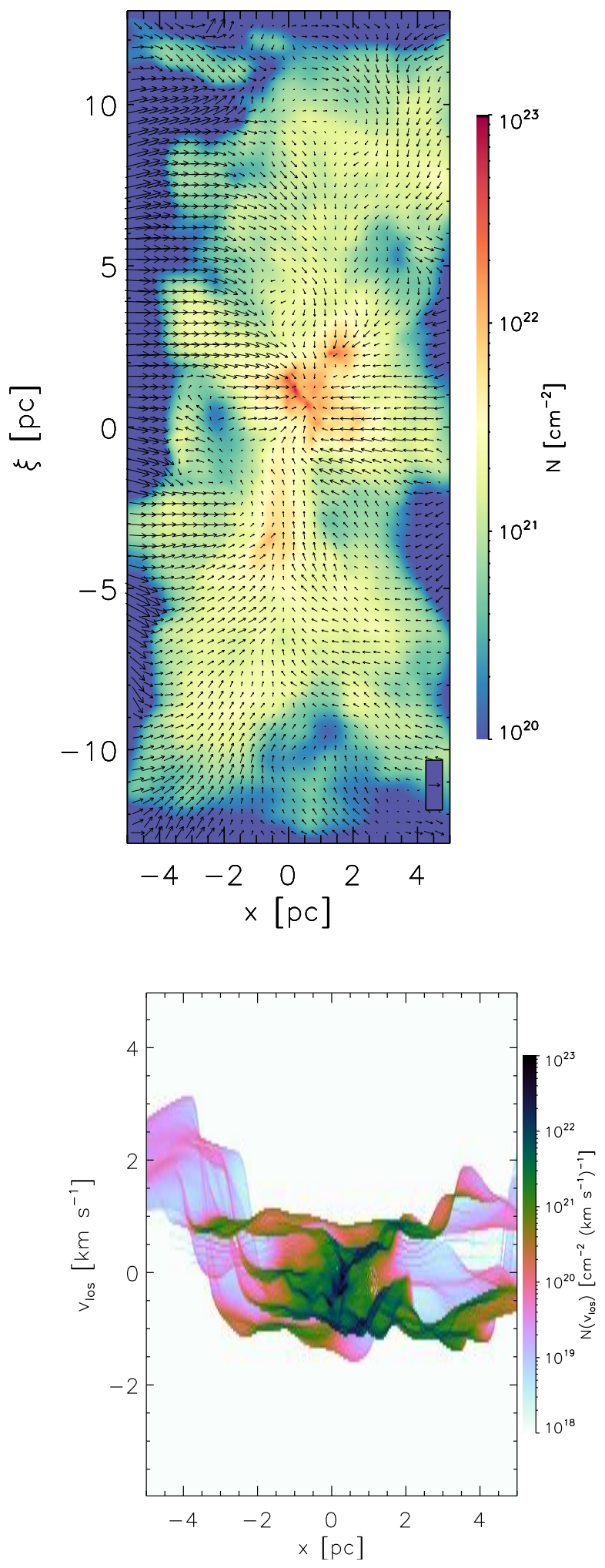

Fig. 15. Simulation results corresponding to data first published in Gómez \& Vázquez-Semadeni (2014). Top panel: column density projection of the simulations at a time step of $21.5 \mathrm{Myr}$ with velocity vectors showing the gas motions on top. Bottom panel: corresponding positionvelocity cut along the $X$-axis at a $Y$-offset of $0.1 \mathrm{pc}$. The color-coding in both plots shows the column density of the gas.

positive offsets there is still some lower column density gas left at positive velocities. While the magnitude of the velocity difference between the two sides of the cloud is smaller than in our observations, qualitatively, the position-velocity cuts of the simulations and observations resemble each other closely.

Since here we are comparing only one simulation with one observational dataset, deriving general conclusions is difficult. However, the qualitative agreement between the observations and simulations indicates that the G28.3 cloud may indeed form via gravitationally driven converging gas flows.

Another, maybe more speculative aspect of these simulations is that at large scales, a phenomenon similar to the inside-out collapse operates (see Sect. 7.2 of Vázquez-Semadeni et al. 2019). That is, there seems to be an expanding infall wave, so that material farther outside begins to fall in later. We speculate that this behavior could also explain the apparent decoupling of the HI from the denser molecular gas in our observational data.

\subsection{Dynamical star formation}

How can we interprete the various results obtained above in the framework of dynamical star formation? Synthesizing the results from the previous sections, we find the following:

- The dense infrared dark cloud is embedded in an extended cloud of more diffuse molecular and atomic gas. While the CNM traced by the HISA does not show any clear velocity gradient, the other three tracers ${ }^{13} \mathrm{CO}(1-0)$, [CI], and ${ }^{13} \mathrm{CO}(3-2)$ exhibit a velocity gradient from north to south. The HOG and velocity structure function analysis confirm this difference indicating a decoupling of the atomic CNM from the denser central cloud. This is further supported by the decrease in HISA toward the central filamentary part of the cloud.

- The position-velocity diagrams of ${ }^{13} \mathrm{CO}(1-0)$, [CI], and ${ }^{13} \mathrm{CO}(3-2)$ reveal two velocity components from the north and the south that converge at a velocity of $\sim 78 \mathrm{~km} \mathrm{~s}^{-1}$ of the central infrared dark cloud. This is indicative of a converging gas flow.

- Estimates of the gas flow rate from the cloud edge to the center reveal rather constant values over all scales. This can be interpreted as a constant gas flow from the outside to the central infrared dark cloud. Such a constant flow rate is consistent with a self-similar, gravitationally driven collapse of a cloud.

- Comparing the derived second-order velocity structure functions with those derived from simulated molecular clouds, the data are consistent with gas flows that are dominated by the formation of hierarchical structures and cores.

Taking these results together, the spatial and kinematic signatures obtained toward the infrared dark cloud G28.3 are consistent with converging gas flows that may trigger the formation of the central IRDC and the gravitational collapse of the cloud as a site of active star formation. It is difficult to clearly differentiate between colliding flows that started in the low-density medium, or a cloud-cloud collision picture where two cold dense clouds collide. Nevertheless, since cloud-cloud collisions may be considered as the high-density version of the broader picture of colliding gas flows, both pictures are consistent with a dynamic cloud and star formation scenario.

The remaining follow-up question relates to the origin of such converging gas flows. Are the flows externally triggered, for example due to nearby supernova explosions or to the spiral arm passage of the gas? Or are the flows produced by the large-scale cloud collapse? The constant flow rates from large to small spatial scales are consistent with a self-similar, gravitationally driven cloud collapse. Furthermore, the converging gas flow signatures are only seen in the denser gas tracers, whereas 
the lower-density and more external CNM, as traced by HISA, does not exhibit any significant velocity gradient; this can be considered as an additional indication that the gas flow is gravitationally driven in the dense gas region. However, with the given data, external compression as the cause of the converging flow signatures cannot be excluded. It may even be that a combination of external compression and gravitational collapse could explain the data: The external driving by a spiral arm shock or supernova compression may be the starting point of the collapse, which then may rapidly be taken over by gravity. In this picture, externally initiated converging gas flows and global gravitational collapse could be part of the same overall scenario.

\section{Conclusions and summary}

The analysis of the kinematic parameters of the cloud surrounding the IRDC G28.3 reveals clear signatures of two gas flows that converge at the position of the central IRDC. The mass flow appears almost constant from large to small spatial scales. The spectral and spatial signatures of the HISA compared to the other tracers are consistent with a kinematic decoupling of the HISA-traced CNM from the denser gas. Overall, the analysis is in general agreement with hierarchical cloud structures that are dynamically evolving within converging gas flows. The origin of such converging flows is consistent with a self-similar, gravitationally driven collapse of the cloud. However, converging gas flows could also be caused by external drivers, for example spiral arm shocks or supernova-driven shocks. Distinguishing the possible different origins of the gas flows remains a subject for future investigations.

Acknowledgements. The National Radio Astronomy Observatory is a facility of the National Science Foundation operated under cooperative agreement by Associated Universities, Inc. H.B. likes to thank a lot for the inspiring discussions at the Harvard-Heidelberg Workshop on Star Formation (2019) about flow rates and their potential measurements, in particular to Diederik Kruijssen, Hope Chen, Shmuel Bialy and Sümeyye Suri. H.B., Y.W. and J.S. acknowledge support from the European Research Council under the Horizon 2020 Framework Program via the ERC Consolidator Grant CSF-648505. H.B. also acknowledges support from the Deutsche Forschungsgemeinschaft via SFB 881, "The Milky Way System" (sub-projects B1, B2 and B8).

\section{References}

Audit, E., \& Hennebelle, P. 2005, A\&A, 433, 1

Audit, E., \& Hennebelle, P. 2010, A\&A, 511, A76

Banerjee, R., Vázquez-Semadeni, E., Hennebelle, P., \& Klessen, R. S. 2009 MNRAS, 398, 1082

Barnes, P. J., Muller, E., Indermuehle, B., et al. 2015, ApJ, 812, 6

Bergin, E. A., Hartmann, L. W., Raymond, J. C., \& Ballesteros-Paredes, J. 2004, ApJ, 612, 921

Bertram, E., Konstandin, L., Shetty, R., Glover, S. C. O., \& Klessen, R. S. 2015, MNRAS, 446, 3777

Beuther, H., Ragan, S. E., Ossenkopf, V., et al. 2014, A\&A, 571, A53

Beuther, H., Bihr, S., Rugel, M., et al. 2016, A\&A, 595, A32

Bialy, S., Burkhart, B., \& Sternberg, A. 2017, ApJ, 843, 92

Bisbas, T. G., Tanaka, K. E. I., Tan, J. C., Wu, B., \& Nakamura, F. 2017, ApJ, 850,23

Butler, M. J., \& Tan, J. C. 2012, ApJ, 754, 5

Butler, M. J., Tan, J. C., \& Kainulainen, J. 2014, ApJ, 782, L30

Chira, R.-A., Beuther, H., Linz, H., et al. 2013, A\&A, 552, A40

Chira, R. A., Ibáñez-Mejía, J. C., Mac Low, M. M., \& Henning, T. 2019, A\&A, 630, A97

Churchwell, E., Babler, B. L., Meade, M. R., et al. 2009, PASP, 121, 213

Clark, P. C., Glover, S. C. O., Klessen, R. S., \& Bonnell, I. A. 2012, MNRAS, 424, 2599

Clark, P. C., Glover, S. C. O., Ragan, S. E., \& Duarte-Cabral, A. 2019, MNRAS, 486, 4622

Dame, T. M., Hartmann, D., \& Thaddeus, P. 2001, ApJ, 547, 792

Dempsey, J. T., Thomas, H. S., \& Currie, M. J. 2013, ApJS, 209, 8
Dénes, H., McClure-Griffiths, N. M., Dickey, J. M., Dawson, J. R., \& Murray, C. E. 2018, MNRAS, 479, 1465

Dickman, R. L., \& Kleiner, S. C. 1985, ApJ, 295, 479

Dobashi, K., Shimoikura, T., Katakura, S., Nakamura, F., \& Shimajiri, Y. 2019 PASJ, 71, S12

Duarte-Cabral, A., Dobbs, C. L., Peretto, N., \& Fuller, G. A. 2011, A\&A, 528, A50

Elmegreen, B. G. 1993, in Protostars and Planets III, eds. E. H. Levy, \& J. I Lunine (Tucson: University of Arizona Press), 97

Esquivel, A., \& Lazarian, A. 2005, ApJ, 631, 320

Ewen, H. I., \& Purcell, E. M. 1951, Nature, 168, 356

Feng, S., Beuther, H., Zhang, Q., et al. 2016a, ApJ, 828, 100

Feng, S., Beuther, H., Zhang, Q., et al. 2016b, A\&A, 592, A21

Franco, J., \& Cox, D. P. 1986, PASP, 98, 1076

Frerking, M. A., Langer, W. D., \& Wilson, R. W. 1982, ApJ, 262, 590

Fujita, S., Torii, K., Kuno, N., et al. 2019, PASJ, https://doi .org/10.1093/ pasj/psz028

Gibson, S. J., Taylor, A. R., Higgs, L. A., \& Dewdney, P. E. 2000, ApJ, 540, 851

Gibson, S. J., Taylor, A. R., Higgs, L. A., Brunt, C. M., \& Dewdney, P. E. 2005a, ApJ, 626, 195

Gibson, S. J., Taylor, A. R., Higgs, L. A., Brunt, C. M., \& Dewdney, P. E. 2005b, ApJ, 626, 214

Glover, S. C. O., Clark, P. C., Micic, M., \& Molina, F. 2015, MNRAS, 448, 1607

Gómez, G. C., \& Vázquez-Semadeni, E. 2014, ApJ, 791, 124

Hartmann, L., Ballesteros-Paredes, J., \& Bergin, E. A. 2001, ApJ, 562, 852

Haworth, T. J., Tasker, E. J., Fukui, Y., et al. 2015, MNRAS, 450, 10

Haworth, T. J., Glover, S. C. O., Koepferl, C. M., Bisbas, T. G., \& Dale, J. E. 2018, New A Rev., 82, 1

Heiles, C., \& Gordon, M. A. 1975, ApJ, 199, 361

Heiner, J. S., Vázquez-Semadeni, E., \& Ballesteros-Paredes, J. 2015, MNRAS, 452, 1353

Heitsch, F., \& Hartmann, L. 2008, ApJ, 689, 290

Heitsch, F., Burkert, A., Hartmann, L. W., Slyz, A. D., \& Devriendt, J. E. G. 2005, ApJ, 633, L113

Heitsch, F., Slyz, A. D., Devriendt, J. E. G., Hartmann, L. W., \& Burkert, A. 2006, ApJ, 648, 1052

Heitsch, F., Hartmann, L. W., Slyz, A. D., Devriendt, J. E. G., \& Burkert, A. 2008, ApJ, 674, 316

Henshaw, J. D., Caselli, P., Fontani, F., et al. 2013, MNRAS, 428, 3425

Henshaw, J. D., Longmore, S. N., \& Kruijssen, J. M. D. 2016, MNRAS, 463, L122

Heyer, M., \& Dame, T. M. 2015, ARA\&A, 53, 583

HI4PI Collaboration (Ben Bekhti, N., et al.) 2016, A\&A, 594, A116

Inoue, T., Hennebelle, P., Fukui, Y., et al. 2018, PASJ, 70, S53

Jackson, J. M., Rathborne, J. M., Shah, R. Y., et al. 2006, ApJS, 163, 145

Jiménez-Serra, I., Caselli, P., Tan, J. C., et al. 2010, MNRAS, 406, 187

Kainulainen, J., \& Tan, J. C. 2013, A\&A, 549, A53

Kalberla, P. M. W., \& Kerp, J. 2009, ARA\&A, 47, 27

Kalberla, P. M. W., Burton, W. B., Hartmann, D., et al. 2005, A\&A, 440, 775

Kavars, D. W., Dickey, J. M., McClure-Griffiths, N. M., Gaensler, B. M., \& Green, A. J. 2005, ApJ, 626, 887

Keene, J. 1995, in Lecture Notes in Physics, The Physics and Chemistry of Interstellar Molecular Clouds, eds. G. Winnewisser, \& G. C. Pelz (Berlin: Spriger), 459,186

Kerp, J., Winkel, B., Ben Bekhti, N., Flöer, L., \& Kalberla, P. M. W. 2011, Astron. Nachr., 332, 637

Kobayashi, M. I. N., Kobayashi, H., Inutsuka, S.-i., \& Fukui, Y. 2018, PASJ, 70, S59

Koyama, H., \& Inutsuka, S.-I. 2000, ApJ, 532, 980

Krčo, M., Goldsmith, P. F., Brown, R. L., \& Li, D. 2008, ApJ, 689, 276

Krumholz, M. R., McKee, C. F., \& Tumlinson, J. 2008, ApJ, 689, 865

Krumholz, M. R., McKee, C. F., \& Tumlinson, J. 2009, ApJ, 693, 216

Langer, W. D., Velusamy, T., Morris, M. R., Goldsmith, P. F., \& Pineda, J. L. 2017, A\&A, 599, A136

Li, D. \& Goldsmith, P. F. 2003, ApJ, 585, 823

Li, G.-X. 2018, MNRAS, 477, 4951

Mac Low, M., \& Klessen, R. S. 2004, Rev. Mod. Phys., 76, 125

McClure-Griffiths, N. M., Pisano, D. J., Calabretta, M. R., et al. 2009, ApJS, 181, 398

McKee, C. F., \& Ostriker, E. C. 2007, ARA\&A, 45, 565

Miesch, M. S., \& Bally, J. 1994, ApJ, 429, 645

Motte, F., Nguyên Luong, Q., Schneider, N., et al. 2014, A\&A, 571, A32 
Murray, C. E., Stanimirović, S., Goss, W. M., et al. 2018, ApJS, 238, 14 Offner, S. S. R., Bisbas, T. G., Bell, T. A., \& Viti, S. 2014, MNRAS, 440 L81

Ossenkopf, V., \& Mac Low, M.-M. 2002, A\&A, 390, 307

Ossenkopf, V., Ormel, C. W., Simon, R., Sun, K., \& Stutzki, J. 2011, A\&A, 525, A9+

Papadopoulos, P. P., Thi, W. F., \& Viti, S. 2004, MNRAS, 351, 147

Pillai, T., Wyrowski, F., Carey, S. J., \& Menten, K. M. 2006, A\&A, 450, 569

Ragan, S., Henning, T., Krause, O., et al. 2012, A\&A, 547, A49

Riegel, K. W., \& Crutcher, R. M. 1972, A\&A, 18, 55

Riener, M., Kainulainen, J., Beuther, H., et al. 2020, A\&A, 633, A14

Rigby, A. J., Moore, T. J. T., Plume, R., et al. 2016, MNRAS, 456, 2885

Rohlfs, K., \& Wilson, T. L. 2006, Tools of Radio Astronomy, eds. K. Rohlfs, \& T. L. Wilson (Berlin: Springer)

Schilke, P., Keene, J., Le Bourlot, J., Pineau des Forets, G., \& Roueff, E. 1995, A\&A, 294, L17

Schuller, F., Menten, K. M., Contreras, Y., et al. 2009, A\&A, 504, 415

Schuller, F., Csengeri, T., Urquhart, J. S., et al. 2017, A\&A, 601, A124

Shimajiri, Y., Sakai, T., Tsukagoshi, T., et al. 2013, ApJ, 774, L20

Soler, J. D., Beuther, H., Rugel, M., et al. 2019, A\&A, 622, A166

Sternberg, A., Le Petit, F., Roueff, E., \& Le Bourlot, J. 2014, ApJ, 790, 10

Stil, J. M., Taylor, A. R., Dickey, J. M., et al. 2006, AJ, 132, 1158
Störzer, H., Stutzki, J., \& Sternberg, A. 1997, A\&A, 323, L13

Tackenberg, J., Beuther, H., Henning, T., et al. 2014, A\&A, 565, A101

Tan, J. C., Kong, S., Butler, M. J., Caselli, P., \& Fontani, F. 2013, ApJ, 779, 96

Tan, J. C., Kong, S., Zhang, Y., et al. 2016, ApJ, 821, L3

Umemoto, T., Minamidani, T., Kuno, N., et al. 2017, PASJ, 69, 78

van der Werf, P. P., Goss, W. M., \& Vanden Bout, P. A. 1988, A\&A, 201, 311

Vázquez-Semadeni, E., Ryu, D., Passot, T., González, R. F., \& Gazol, A. 2006, ApJ, 643, 245

Vázquez-Semadeni, E., Banerjee, R., Gómez, G. C., et al. 2011, MNRAS, 414, 2511

Vázquez-Semadeni, E., Palau, A., Ballesteros-Paredes, J., Gómez, G. C., \& Zamora-Avilés, M. 2019, MNRAS, 490, 3061

Wang, Y., Zhang, Q., Pillai, T., Wyrowski, F., \& Wu, Y. 2008, ApJ, 672, L33

Wang, Y., Beuther, H., Rugel, M. R., et al. 2020a, A\&A, 634, A83

Wang, Y., Bihr, S., Beuther, H., et al. 2020b, A\&A, 634, A139

Whitworth, A., \& Summers, D. 1985, MNRAS, 214, 1

Wienen, M., Wyrowski, F., Schuller, F., et al. 2012, A\&A, 544, A146

Williams, J. P., Blitz, L., \& McKee, C. F. 2000, Protostars and Planets IV (Tucson: University of Arizona Press), 97

Winkel, B., Kerp, J., Flöer, L., et al. 2016, A\&A, 585, A41

Zhang, Q., Wang, K., Lu, X., \& Jiménez-Serra, I. 2015, ApJ, 804, 141 